\title{
Infinite Dimensional Groups and Riemann Surface Field Theories
}

\author{
A.L. Carey ${ }^{1}$, K.C. Hannabuss ${ }^{2}$ \\ ${ }^{1}$ Department of Pure Mathematics, University of Adelaide, Adelaide 5005, Australia \\ 2 Balliol College, Oxford, U.K.
}

Received: 4 February 1994/in revised form: 10 April 1995

\begin{abstract}
We show how to obtain positive energy representations of the group $\mathscr{G}$ of smooth maps from a union of circles to $U(N)$ from geometric data associated with a Riemann surface having these circles as boundary. Using covering spaces we can reduce to the case where $N=1$. Then our main result shows that Mackey induction may be applied and yields representations of the connected component of the identity of $\mathscr{G}$ which have the form of a Fock representation of an infinite dimensional Heisenberg group tensored with a finite dimensional representation of a subgroup isomorphic to the first cohomology group of the surface obtained by capping the boundary circles with discs. We give geometric sufficient conditions for the correlation functions to be positive definite and derive explicit formulae for them and for the vacuum (or cyclic) vector. (This gives a geometric construction of correlation functions which had been obtained earlier using tau functions.) By choosing particular functions in $\mathscr{G}$ with non-zero winding numbers on the boundary we obtain analogues of vertex operators described by Segal in the genus zero case. These special elements of $\mathscr{G}$ (which have a simple interpretation in terms of function theory on the Riemann surface) approximate fermion (or Clifford algebra) operators. They enable a rigorous derivation of a form of boson-fermion correspondence in the sense that we construct generators of a Clifford algebra from the unitaries representing these elements of $\mathscr{G}$.
\end{abstract}

\section{Introduction}

The aim of this paper is to show how geometric data associated to a Riemann surface lead naturally to unitary representations of infinite dimensional Lie groups and representations of Clifford and Heisenberg algebras. Our study is related to an extensive earlier literature. However, we have attempted to make our discussion comparatively self contained.

Initially we were motivated by a desire to understand some of the literature on conformal quantum field theory [A-GMV, A-GNMV, A-GBNMV, E]. Significant progress in this direction has come from the algebraic approach of [DJKM, KNTY]. The starting point of the latter is the so-called tau function and its relation to soliton 
hierarchies. The language of these papers is that of vector spaces of formal power series, infinite order differential operators (vertex operators) and representations of Kac-Moody and Virasoro algebras. A more fundamental viewpoint is that of Segal [S2] in which he postulates an axiomatic framework for conformal field theories where these vector spaces are endowed with an inner product and the vertex operators are re-interpreted in terms of representations of loop groups, diffeomorphism groups, Clifford algebras and intertwining operators. These latter objects replace the Lie algebraic viewpoint of the earlier work. Our aim in this paper is to provide some explicit examples of this more geometric and global approach. In particular we show how to obtain representations of the type considered in [KNTY] from the following geometric data. We first recall that for any smooth oriented 1-manifold $S$ with a spin structure $\lambda$ (that is a real line bundle such that $\lambda \otimes \lambda=T^{*} S$ ) the real space $\mathscr{K}_{\mathbf{R}}=\Gamma(S, \lambda)$ has a quadratic form which pairs sections $\alpha_{1}$ and $\alpha_{2}$ to give

$$
\left(\alpha_{1}, \alpha_{2}\right)=\int_{S} \alpha_{1} \otimes \alpha_{2}
$$

The corresponding complex Clifford $*$-algebra $C\left(\mathscr{K}_{\mathbf{R}}\right)$ has a unique irreducible *-representation with positive energy for any parametrisation of $S$. When $S$ is the boundary of a Riemann surface $\Sigma_{1}$ on which there is a complex line bundle $L_{1}$ whose restriction to $S$ is $\lambda \otimes \mathbf{C}$, the space $\mathscr{H}$ on which this representation acts is given by $C\left(\mathscr{K}_{\mathbf{R}}\right) / \mathscr{J}$, where $\mathscr{J}$ is the left ideal generated by $\mathscr{K}_{1}=\Gamma\left(\Sigma_{1}, L_{1}\right)$. Finally, suppose that we have a Riemann surface $\Sigma$ and a decomposition $\Sigma=\Sigma_{1} \cup \Sigma_{2}$ into two submanifolds which intersect in their common smooth boundary, $\partial \Sigma_{1}=$ $S=\partial \Sigma_{2}$, and a line bundle $L$ over $\Sigma$ such that $\left.L\right|_{\Sigma_{j}}=L_{j}$. The decomposition of $\Sigma$ into submanifolds naturally defines a decomposition of $\mathscr{K}=\mathscr{K}_{1} \oplus \mathscr{K}_{2}$ into subspaces isotropic with respect to the bilinear form and this data in turn defines a Fock representation of the Clifford algebra on the exterior algebra over $\mathscr{K}_{1}$. This space is isomorphic to the irreducible $*$-representation space $\mathscr{H}$, but the identification is only unitary when $\Sigma$ is the Schottky double of $\Sigma_{1}$ and the line bundle $L$ is compatible with the Schottky involution.

The first two sections follow this approach, but in the case of the Schottky double it is more natural to replace the real Clifford algebras by Araki's self-conjugate CAR formalism, [Ar], and this is done from Sect. 3 onwards. Given a complex line bundle $L$ on $\Sigma$ compatible with the Schottky involution, $\bar{L} \otimes L$ is the complexification of $T^{*} S$ so that there is a pairing between $\mathscr{K}_{1}$ and $\mathscr{K}_{2}=\overline{\mathscr{K}_{1}}$ and each is an isotropic subspace of $\mathscr{K}=\mathscr{K}_{1} \oplus \mathscr{K}_{2}$. The group $\mathscr{G}$ of analytic $U(1)$ valued maps on $\partial \Sigma_{1}$ acts on $\mathscr{K}_{j}$ by multiplication and so defines a group of automorphisms of the Clifford algebra $C(\mathscr{K})$. There are implementible so that a central extension of $\mathscr{G}$ has a representation $\Gamma$ on $\mathscr{H}$. At the Lie algebra level one obtains a representation of a Heisenberg algebra thus generalising the paper [JKL].

The main interest in conformal field theory is in the properties of the representation of $\mathscr{G}$ (or more generally in the groups of smooth compact Lie group valued functions on $\partial \Sigma_{1}$, see Sect. 6). One of the main results of our work is a proof in Sect. 3 and 4, that when $\Sigma$ is a Schottky double (using standard tools of representation theory due to Mackey together with earlier results of Segal [S1] and Carey, Ruijsenaars and Palmer [CR, CP]), this representation is cyclic (in fact irreducible), with cyclic vector $\Omega$ say, and that one may explicitly compute the "matrix elements"

$$
\langle\Omega, \Gamma(g) \Omega\rangle, \quad g \in \mathscr{G}
$$


The resulting formulae imply those involving the tau-functions of [KNTY] at least in certain cases. In Sect. 2 we indicate how one should interpret [KTNY] when $\Sigma$ is not a Schottky double although we do not derive explicit formulae. (Special cases of the results of Sect. 2 to 4 were announced in [CHM].)

In $[\mathrm{CHM}]$ we sketched how the boson-fermion correspondence should appear in this framework as a relationship between the projective representation of $\mathscr{G}$ and the Clifford algebra. In the present paper we prove an explicit form of this correspondence in the case where $\Sigma$ is a Schottky double. Our proof differs from previous work in a number of ways. First, it starts from a more fundamental representation theoretic viewpoint (for example in [KNTY] the correspondence is more or less built in by the assumption that the tau function is the generating function for both the Heisenberg and Clifford algebras). In our approach the starting point is the geometric data described above. This leads to a geometrically defined inner product on the exterior algebra and hence to unitary group representations on Hilbert spaces such that the correlation functions satisfy Wightman positivity. The latter is essential for a bona fide quantum field theory. When one does not have a Schottky double we know how to interpret these correlation functions (Sect. 2), however they no longer satisfy positivity and we do not investigate the meaning of the boson-fermion correspondence in this generality. Another interpretation of these correlation functions is given in [R1, R2]. The second point of particular interest in our approach is the discovery of a generalisation of Segal's vertex operators [S1] (for genus zero) to surfaces of arbitrary genus.

In Sect. 6 we investigate higher rank bundles over Riemann surfaces on which one has an action of the group of smooth maps from $\partial \Sigma_{1}$ to a compact Lie group $G$. We consider the case $G=U(N)$ in detail by using a covering space argument similar to that of [SW]. We thus reduce the higher rank case to that of line bundles dealt with in the earlier sections. Again we indicate how to interpret Segal's vertex operators for higher rank bundles over curves of arbitrary genus. In Sect. 7 we investigate how the KMS condition in genus one [CH1] generalises to higher genus surfaces.

We should like to thank the referee for some helpful comments and in particular for suggesting a better proof of Lemma 3.3 and the geometric interpretation of $\mathscr{W}$ which follows it.

\section{Fermions on a Riemann Surface}

We shall follow the algebraic description of fermions presented in $[\mathrm{CEH}]$ and [CHM], which for convenience we recall here. Let $L$ be a line bundle over a Riemann surface $\Sigma$, and suppose that the surface has an open covering by two sets $U_{1}$ and $U_{2}$. Writing $\Gamma(\Sigma, \mathcal{O}(L))$ for the global sections of the sheaf $\mathcal{O}(L)$ of germs of holomorphic sections of $L$ and $H^{1}(\Sigma, O(L))$ for the first cohomology group with coefficients in the sheaf, the Mayer-Vietoris sequence can be written as

$$
\begin{aligned}
0 & \rightarrow \Gamma(\Sigma, \mathcal{O}(L)) \rightarrow \Gamma\left(U_{1}, \mathcal{O}(L)\right) \oplus \Gamma\left(U_{2}, \mathcal{O}(L)\right) \rightarrow \Gamma\left(U_{1} \cap U_{2}, \mathcal{O}(L)\right) \\
& \rightarrow H^{1}(\Sigma, \mathcal{O}(L)) \rightarrow 0
\end{aligned}
$$

In the case of fermions we choose $L$ to be an even spin structure for which $\Gamma(\Sigma, \mathcal{O}(L))$ vanishes (as happens generically, $[\mathrm{F}]$ ). By Serre duality $H^{1}(\Sigma, \mathcal{O}(L)$ ) 
also vanishes and the sequence reduces to

$$
0 \rightarrow \Gamma\left(U_{1}, \mathcal{O}(L)\right) \oplus \Gamma\left(U_{2}, \mathcal{O}(L)\right) \rightarrow \Gamma\left(U_{1} \cap U_{2}, \mathcal{O}(L)\right) \rightarrow 0
$$

from which we deduce that there is a decomposition

$$
\Gamma\left(U_{1} \cap U_{2}, \mathcal{O}(L)\right)=\Gamma\left(U_{1}, \mathcal{O}(L)\right) \oplus \Gamma\left(U_{2}, \mathcal{O}(L)\right) .
$$

Let us now suppose that $\Sigma_{1}$ and $\Sigma_{2}$ are closed submanifolds of $\Sigma$ which intersect in their common smooth boundary

$$
\Sigma_{1} \cap \Sigma_{2}=\partial \Sigma_{1}=\partial \Sigma_{2} .
$$

For $j=1$ and 2 we choose a sequence of neighbourhoods $U_{j}$ which shrink down to $\Sigma_{j}$, so that $\Gamma\left(U_{1} \cap U_{2}, \mathcal{O}(L)\right)$ increases to $\mathscr{K}=\Gamma\left(\Sigma_{1} \cap \Sigma_{2}, \mathcal{O}(L)\right)=\Gamma\left(\partial \Sigma_{1}, \mathcal{O}(L)\right)$. The spaces $\Gamma\left(U_{j}, \mathcal{O}(L)\right)$ increase to give spaces $\mathscr{K}_{j}$ such that

$$
\mathscr{K}=\mathscr{K}_{1} \oplus \mathscr{K}_{2} .
$$

Since $L$ is a spin bundle the tensor product of sections $\alpha_{j} \in \Gamma\left(U_{j}, \mathcal{O}(L)\right)$ gives a section of the canonical bundle $K$. Choosing an orientation of $\partial \Sigma_{1}$ we may integrate $\alpha_{1} \otimes \alpha_{2}$ round the boundary to get a natural symmetric non-degenerate bilinear form on $\mathscr{K}$,

$$
\left(\alpha_{1}, \alpha_{2}\right)=\int_{\partial \Sigma_{1}} \alpha_{1} \otimes \alpha_{2}
$$

If both sections $\alpha_{1}$ and $\alpha_{2}$ have holomorphic extensions to $U_{1}$ (or $U_{2}$ ) then their product also extends and by Cauchy's theorem the integral defining $\left(\alpha_{1}, \alpha_{2}\right)$ vanishes. From this we deduce that $\Gamma\left(U_{j}, \mathcal{O}(L)\right)$ and its limit $\mathscr{K}_{j}$ are isotropic, for $j=1$ or 2. It is easy to see that (1.1) is a non-degenerate bilinear form on $\mathscr{K}$ and therefore defines a pairing of the subspaces $\mathscr{K}_{1}$ and $\mathscr{K}_{2}$.

Any decomposition of an inner product space into isotropic subspaces

$$
\mathscr{K}=\mathscr{K}_{1} \oplus \mathscr{K}_{2},
$$

gives rise ${ }^{+} \mathrm{O}$ a natural representation $\Psi_{21}$ of the Clifford algebra of $\mathscr{K}$ on the exterior algebra $\wedge \mathscr{K}_{1}$. Elements $\alpha$ of $\mathscr{K}_{1}$ act by exterior multiplication,

$$
\Psi_{21}(\alpha): \alpha_{1} \wedge \alpha_{2} \wedge \cdots \wedge \alpha_{r} \mapsto \alpha \wedge \alpha_{1} \wedge \alpha_{2} \wedge \cdots \wedge \alpha_{r}
$$

whilst elements of $\mathscr{K}_{2}$ act by inner multiplication,

$$
\Psi_{21}(\alpha): \alpha_{1} \wedge \alpha_{2} \wedge \cdots \wedge \alpha_{r} \mapsto \sum_{k=1}^{r}(-1)^{k-1}\left(\alpha, \alpha_{k}\right) \wedge \alpha_{1} \wedge \alpha_{2} \cdots \wedge \alpha_{k-1} \wedge \alpha_{k+1} \cdots \wedge \alpha_{r}
$$

(The pairing of the isotropic subspaces $\mathscr{K}_{1}$ and $\mathscr{K}_{2}$ extends to their exterior algebras and the inner multiplication action of $\mathscr{K}_{2}$ is just the transpose of exterior multiplication on $\wedge \mathscr{K}_{2}$.) These conditions determine $\Psi_{21}$ and ensure the usual relations

$$
\Psi_{21}(\beta) \Psi_{21}(\alpha)+\Psi_{21}(\alpha) \Psi_{21}(\beta)=(\beta, \alpha),
$$

for all $\beta$ and $\alpha$ in $\mathscr{K}_{1} \oplus \mathscr{K}_{2}$.

For $j=1$ or 2 , there is a cyclic vector $\Omega_{j}=1 \oplus 0 \oplus 0 \cdots \in \wedge \mathscr{K}_{j}$, called the vacuum vector. With respect to the pairing of $\wedge \mathscr{K}_{1}$ and $\wedge \mathscr{K}_{2}, \Psi_{21}$ and $\Psi_{12}$ are dual representations of the Clifford algebra.

We summarise the discussion above. 
Proposition 1.1. Associated to every decomposition of a Riemann surface $\Sigma$ as the union of submanifolds $\Sigma_{1}$ and $\Sigma_{2}$ with common boundary and a generic even spin structure $L$ over $\Sigma$, we have the following data:

(i) A non-degenerate bilinear form (1.1) on the real analytic sections $\mathscr{K}$ of $L$ restricted to $\Sigma_{1} \cap \Sigma_{2}$.

(ii) A Fock representation of the Clifford algebra over $\mathscr{K}$ defined by (1.1) on the exterior algebra over the space of sections of $L$ restricted to either $\Sigma_{1}$ or $\Sigma_{2}$. These representations are dual to each other.

We now introduce the (not necessarily orthogonal) projection $P_{k j}$ onto $\mathscr{K}_{j}$ along $\mathscr{K}_{k}$. Since $\mathscr{K}_{1}$ and $\mathscr{K}_{2}$ are isotropic we have

$$
\left(\beta, P_{21} \alpha\right)=\left(P_{12} \beta, P_{21} \alpha\right)=\left(P_{12} \beta, \alpha\right),
$$

so that $P_{21}$ and $P_{12}$ are transpose maps with respect to the bilinear form. It follows from the definition of $\Psi_{21}$ that

$$
\left(\Omega_{2}, \Psi_{21}(\beta) \Psi_{21}(\alpha) \Omega_{1}\right)=\left(\beta, P_{21} \alpha\right)
$$

Given another decomposition $\mathscr{K}=\mathscr{K}_{3} \oplus \mathscr{K}_{2}$, there is a natural map $T_{13}^{2}$ from $\wedge \mathscr{K}_{3}$ to $\wedge \mathscr{K}_{1}$ which maps $\Omega_{3}$ to $\Omega_{1}$ and intertwines the Clifford algebra representations $\Psi_{23}$ and $\Psi_{21}$, which is defined by

$$
T_{13}^{2} \Psi_{23}(\alpha) \Omega_{3}=\Psi_{21}(\alpha) \Omega_{1} .
$$

This is well-defined since $\Psi_{23}(\alpha) \Omega_{3}$ vanishes if and only if $\alpha$ is in the ideal generated by $\mathscr{K}_{2}$ and then $\Psi_{21}(\alpha) \Omega_{1}$ vanishes too.

The normal arena for quantum field theory is a Hilbert space, which, by the Riesz representation theorem, means that there is an antilinear identification of the space and its dual. Such an antilinear map arises naturally from the geometry if one takes $\Sigma$ to be a Schottky double (cf. [JKL, CH1]) with its natural antiholomorphic involution taking $z \in \Sigma_{1}$ to the corresponding point $\tilde{z}$ in $\Sigma_{2}$ (thus fixing each point of the boundary). Thus, as a real manifold, $\Sigma_{2}$ is an oppositely oriented copy of $\Sigma_{1}$. For more on Schottky doubles, see $[\mathrm{F}, \mathrm{H}]$.

Proposition 1.2. Let $\Sigma$ be a Schottky double.

(i) The Schottky involution induces maps of forms and $\frac{1}{2}$-forms, written for brevity as $\alpha(z) \mapsto \alpha(\tilde{z})$. The image is an antiholomorphic $\frac{1}{2}$-form, so that its complex conjugate is holomorphic.

(ii) Defining $\tilde{\alpha}(z)=\overline{\alpha(\tilde{z})}$, we obtain an antilinear map, $\sim$ with

(i.e. $\sim$ is antiorthogonal).

$$
(\tilde{\alpha}, \tilde{\beta})=\int_{\partial \Sigma_{1}} \overline{\alpha(z) \beta(z)}=\overline{(\alpha, \beta)}
$$

(iii) The map in (ii) satisfies $(\tilde{\alpha}, \alpha)=\int_{\partial \Sigma_{1}}|\alpha|^{2}$, and hence $\langle\alpha, \beta\rangle=(\tilde{\alpha}, \beta)$ defines an inner product on $\mathscr{K}$.

(iv) There is a natural isomorphism of the Clifford algebra over $\mathscr{K}$ with the fermion algebra (or algebra of the canonical anticommutation relations: CAR) over $\mathscr{K}$ regarded as a pre-Hilbert space in this inner product.

Proof. The statements (i),(ii) and (iii) are clear. For (iv) observe that in general whenever there is an antiorthogonal involution $\sim$ which maps $\mathscr{K}_{1}$ to $\mathscr{K}_{2}$ (where 
$\left.\mathscr{K}=\mathscr{K}_{1} \oplus \mathscr{K}_{2}\right)$ and vice versa, and such that $(\tilde{\alpha}, \alpha)>0$ for all non-zero $\alpha$ one can define an inner product

$$
\langle\alpha, \beta\rangle=(\tilde{\alpha}, \beta)
$$

on $\mathscr{K}$ and, in particular, on the subspace $\mathscr{K}_{1}$. The map $\sim$ and this inner product both extend to $\wedge \mathscr{K}_{1}$, which can then be completed to give a Hilbert space $\mathscr{F}_{1}{ }^{\circ}$. In this situation we drop one suffix and write $P_{1}, \Psi_{1}$ instead of $P_{21}$ and $\Psi_{21}$.

To complete the proof let $\alpha \in \mathscr{K}_{1}$ and $\phi \in \wedge \mathscr{K}_{1}$. Then we have

$$
\begin{aligned}
\langle\Psi(\alpha) \phi, \psi\rangle & =\langle\alpha \wedge \phi, \psi\rangle=(\tilde{\alpha} \wedge \tilde{\phi}, \psi) \\
& =(\tilde{\phi}, \Psi(\tilde{\alpha}) \psi)=\langle\phi, \Psi(\tilde{\alpha}) \psi\rangle .
\end{aligned}
$$

We therefore deduce the important relation that for $\alpha \in \mathscr{K}_{1}$,

$$
\Psi(\alpha)^{*}=\Psi(\tilde{\alpha}) .
$$

Applying $\sim$ the same applies for $\alpha \in \mathscr{K}_{2}$, but we shall not need that. Thence the Clifford algebra relations can be recast into CAR form as

and

$$
\Psi_{1}(\alpha)^{*} \Psi_{1}(\beta)+\Psi_{1}(\beta) \Psi_{1}(\alpha)^{*}=\langle\alpha, \beta\rangle,
$$

$$
\Psi_{1}(\alpha) \Psi_{1}(\beta)+\Psi_{1}(\beta) \Psi_{1}(\alpha)=0,
$$

for all $\alpha$ and $\beta$ in $\mathscr{K}_{1}$. This completes the proof but in fact more is true.

Setting $\phi=\Omega_{1}$ and $\psi=\Psi(\beta) \Omega_{1}$ in the preceding proof, the formula for the two point correlation function can be recast as

$$
\left\langle\Omega_{1}, \Psi(\alpha)^{*} \Psi(\beta) \Omega_{1}\right\rangle=\left(\tilde{\alpha}, P_{1} \beta\right)=\left\langle\alpha, P_{1} \beta\right\rangle,
$$

which is the usual formula for the Fock space correlations. From this, or by direct calculation, one also can deduce that $P_{1}=P_{1}^{*}$, so that $P_{1}$ is an orthogonal projection.

Lemma 1.3. [CHM] The projection $P_{1}$ is given by an integral operator. Its kernel is the Szegö kernel, $\Lambda$, which can be written explicitly in terms of the theta function $\theta[e]$ associated to the same even half-period $e$ which specifies the choice of spin bundle $L$, and the Schottky-Klein prime form $E$, which is a- $\frac{1}{2}$-form in each of its arguments:

$$
\Lambda(x, y)=\frac{\theta[e](y-x)}{2 \pi i \theta[e](0) E(y, x)} .
$$

This formula makes it clear that $\Lambda$ can be defined for any surface, $\Sigma$ whether or not it is a Schottky double, and we shall see later that there is a more general formula for the correlation functions which also works outside the Hilbert space setting.

\section{Equivalence of Representations}

There is a special case of the preceding situation for which more detailed information is available. Henceforth we assume, following Segal [S2], that the boundary of $\Sigma_{1}$ consists of parametrised circles (we make this assumption precise in our next result). 
Lemma 2.1. Assume there are coordinate charts containing each boundary circle such that in terms of a local coordinate $z,|z|=1$ is the boundary circle. Then the Hilbert space representations of the CAR defined by different Riemann surfaces $\Sigma_{1}$ and spin bundles $L$ which have the same boundary $\partial \Sigma_{1}$ and restriction $L \mid \partial \Sigma_{1}$ which we constructed in Proposition 1.1 are all equivalent.

This is proved in [PS, Sect. 8.11], but only in the case of a single boundary component and a particular spin structure, so in this section we shall outline the modifications needed to prove the lemma in the general case.

The spin bundle can be trivialised in such a way that its sections can be identified either with functions on the circle or with functions multiplied by $z^{1 / 2}$. Thus square-integrable sections are either identified with $L^{2}\left(S^{1}\right)$ or with $z^{1 / 2} L^{2}\left(S^{1}\right)$. Just as there is a standard polarisation of $L^{2}\left(S^{1}\right)$ into the two Hardy spaces $H_{+}$and $H_{-}$, so $z^{1 / 2} L^{2}\left(S^{1}\right)$ can be polarised into $z^{1 / 2} H_{+}$and $z^{1 / 2} H_{-}$. We shall show that the representation defined on $\mathscr{F}_{1}$ using the decomposition into holomorphic sections on $\Sigma_{1}$ and its reflection $\Sigma_{4}=\phi\left(\Sigma_{1}\right)$ is equivalent to that defined by using the appropriate Hardy space decomposition of the sections of $\left.L\right|_{\partial \Sigma_{1}}$, and so that all such representations are equivalent.

Let us first concentrate on a single boundary circle. Since the two spin bundles correspond under multiplication by $z^{1 / 2}$, we need only consider the case of $L^{2}\left(S^{1}\right)$. We cap the circle with a disc and suppose the local coordinate chosen in such a way that $|z| \geqq 1$ gives the disc and points of $\Sigma_{1}$ have $|z| \leqq 1$. There is an injection, $I$ of the boundary values of holomorphic sections on $\Sigma_{1}$ into $L^{2}\left(S^{1}\right)$, and projections $p_{+}$and $p_{-}$onto the two Hardy spaces. The first thing to be checked is that $p_{-} I$ is a Hilbert Schmidt operator. Now $p_{-}$commutes with the dilation operators $R_{\rho}$ defined by

$$
\left(R_{\rho} f\right)(z)=f\left(\rho^{-1} z\right),
$$

so that $p_{-} I=R_{\rho} p_{-} R_{\rho}^{-1} I$. As in [PS], one sees that, for $\rho \in(0,1), p_{-} R_{\rho}^{-1} I$ is bounded. Since, for such $\rho, R_{\rho}$ is trace-class on $H_{-}$, we see that $p_{-} I$ is traceclass, and so Hilbert-Schmidt. Finally, the map $I$ is an isomorphism onto its range so that $I=p_{+} I+p_{-} I=p_{+} I+$ compact. Hence $p_{+} I: W \mapsto I W$ differs from an invertible operator by a compact operator and hence is Fredholm, which shows that the representation defined by $\Sigma_{1}$ is equivalent to the usual one defined by the Hardy spaces $H_{ \pm}$.

Remark. Having established this equivalence with the standard representation, we know (see [PS]) that the existence of the equivalence does not depend on the precise choice of holomorphic local coordinate as the group $\operatorname{Diff}\left(S^{1}\right)$ acts in the Hilbert space of this standard representation (enabling us to change parametrisation).

In the physics literature it is not usual to assume that the Riemann surface is a Schottky double. Nevertheless fermion correlation functions are written down for the representations defined by the Krichever map (see Segal and Pressley for a discussion of the latter). To understand what these correlation functions mean we need to extend the preceding discussion.

In understanding the Krichever map it is useful to compare the theory obtained by capping $\Sigma_{1}$ by its Schottky dual $\Sigma_{2}$ with that obtained when one caps it with another space $\Sigma_{-}$to give a closed surface. To do this we need to suppose that $\Sigma_{-}$ is the Schottky dual of $\Sigma_{+}$. We now have three different ways of decomposing $\mathscr{K}$ :

$$
\mathscr{K}=\mathscr{K}_{1} \oplus \mathscr{K}_{2}=\mathscr{K}_{1} \oplus \mathscr{K}_{-}=\mathscr{K}_{+} \oplus \mathscr{K}_{-} .
$$


The first and third of these define Fock representations $\Psi_{+}$and $\Psi_{1}$ which, by Lemma 2.1, are intertwined by some unitary operator $U$. Denoting transpose by $T$ and using our earlier definitions we have

$$
\begin{aligned}
\left(\Omega_{2}, \Psi_{1}(\alpha) U \Omega_{+}\right) & =\left(T_{2-}^{1} \Omega_{-}, \Psi_{21}(\alpha) U \Omega_{+}\right)=\left(\Omega_{-},\left(T_{2-}^{1}\right)^{\top} \Psi_{12}(\alpha)^{\top} U \Omega_{+}\right) \\
& =\left(\Omega_{-}, \Psi_{1-}(\alpha)^{\top}\left(T_{2-}^{1}\right)^{\top} U T_{+1}^{2} \Omega_{1}\right) .
\end{aligned}
$$

Now, from the earlier equivalences, $\left(T_{2-}^{1}\right)^{\top} U T_{+1}^{2}$ intertwines $\Psi_{-1}=\Psi_{1-}^{\top}$ with itself and so, by Schur's Lemma, is a multiple, $k$, of the identity, giving

$$
\left(\Omega_{2}, \Psi_{1}(\alpha) U \Omega_{+}\right)=k\left(\Omega_{-}, \Psi_{-1}(\alpha) \Omega_{1}\right) .
$$

Applying the same argument to products in the Clifford algebra now gives

$$
\begin{aligned}
\left\langle\Omega_{1}, \Psi_{1}(\alpha) \Psi_{1}(\beta) U \Omega_{+}\right\rangle & =\left(\Omega_{2}, \Psi_{1}(\alpha) \Psi_{1}(\beta) U \Omega_{+}\right)=k\left(\Omega_{-}, \Psi_{-1}(\alpha) \Psi_{-1}(\beta) \Omega_{1}\right) \\
& =k\left(\alpha, P_{-1} \beta\right) .
\end{aligned}
$$

Thus correlation functions involving two different Fock cyclic vectors, $\Omega_{1}$ and $\Omega_{+}$, can also be computed purely in terms of the geometrical projection involving the surface obtained by capping $\Sigma_{1}$ with the Schottky dual of $\Sigma_{+}$.

This provides an interpretation of the correlation functions involved in the Krichever construction where one uses for $\Sigma_{-}$a union of discs. In some papers these correlation functions are misleadingly written as inner products involving the same cyclic vector (i.e. $\Omega_{1}$ is identified with $\Omega_{+}$). Under the construction we have given here this may only be done in the Schottky double case. This is what distinguishes the latter from other possibilities: it is only in the Schottky case that there is a geometrically defined inner product on $\mathscr{K}$ in terms of which the correlation functions are positive definite and hence one can obtain a bona fide (in the sense of satisfying the usual axioms) quantum field theory.

Corollary 2.2. The representation defined by any Riemann surface $\Sigma_{1}$ is equivalent to that obtained simply by capping the $p+1$ circles which make up $\partial \Sigma_{1}$ by discs, that is, it is equivalent to a tensor product of $p+1$ standard fermion representations for a single circle.

\section{The Boson Representation}

In the remaining sections we shall take $\Sigma$ to be the Schottky double formed from $\Sigma_{+}$and with parametrised boundary circles, and we work with the complex selfconjugate CAR formalism of Araki, [Ar]. The group $\mathscr{G}=\operatorname{Map}\left(\partial \Sigma_{+}, \mathbf{T}\right)$ of smooth functions from $\partial \Sigma_{+}$to the complex numbers of modulus 1 acts orthogonally by pointwise multiplication on $\mathscr{K}=L^{2}\left(\partial \Sigma_{+}\right)$. This action extends to an automorphism of the Clifford algebra so that for $\xi \in \mathscr{G}$ and $\alpha \in \mathscr{K}$ we have

$$
\xi: \Psi(\alpha) \mapsto \Psi(\xi \cdot \alpha) \text {. }
$$

We will refer to $\mathscr{G}$ somewhat loosely as the "bosons" even though strictly speaking it is the Lie algebra of this group which can be given the structure of a Heisenberg algebra and hence may be regarded as representing bosons.

As $\mathscr{G}$ is the product of groups of smooth maps on each connected component of the boundary and the representation $\Psi$ is equivalent to the standard representation 
obtained by capping the boundary by discs (Lemma 2.1), this automorphism is implemented by an irreducible projective representation $\Gamma$ with 2-cocycle $\sigma$, that is

$$
\begin{aligned}
\Psi(\xi \cdot \alpha) & =\Gamma(\xi) \Psi(\alpha) \Gamma(\xi)^{-1}, \\
\Gamma\left(\xi_{1}\right) \Gamma\left(\xi_{2}\right) & =\sigma\left(\xi_{1}, \xi_{2}\right) \Gamma\left(\xi_{1} \xi_{2}\right) .
\end{aligned}
$$

Definition 3.1. Choose a base point $c_{j}$ on the $j^{\text {th }}$ boundary circle for $j=0,1$, $2, \ldots, p$. Let $\xi \in \mathscr{G}$ and $\xi=e^{i f}$. Define $\Delta_{j} f$ to be the change in the value of $a$ function $f$ after one circuit of that boundary circle. The Lie algebra of $\mathscr{G}$ consists of those $f$ with $\Delta_{j} f=0$ for all $j=0,1,2, \ldots, p$.

Following Segal [S1] we note that there is a choice of unitary $\Gamma(\xi)$ for each $\xi \in G$ such that the cocycle has the form

$$
\sigma\left(e^{i f_{1}}, e^{i f_{2}}\right)=e^{-i s(f, g) / 4 \pi}=\exp \left(-\frac{i}{4 \pi}\left(\int_{\partial \Sigma_{+}} f_{2} d f_{1}+\sum_{j=1}^{p} f_{2}\left(c_{j}\right) \Delta_{j} f_{1}\right)\right) .
$$

Remarks. 3.1.

(i) Although we have not chosen to do so here this cocycle may be derived by a purely geometric argument as in [CHM]. On the Lie algebra of $\mathscr{G}$ the bilinear form $s$ in (3.3) is symplectic, equipping this Lie algebra with the structure of an infinite dimensional Heisenberg algebra.

(ii) In the case where $\Sigma$ has genus one $[\mathrm{CH}]$ we were able to analyse the projective representation $\Gamma$ of $G$ explicitly by exploiting its action as automorphisms and using some special features of that example. In the higher genus case we seem forced to take a more indirect path to an understanding of $\Gamma$.

By using Mackey's subgroup analysis [M1] it is possible to make some general remarks about the representation $\Gamma$ without exploiting its provenance as automorphisms of the fermions. Let us start by defining the two subgroups $\mathscr{T}$ of globally constant functions in $\mathscr{G}$, and $\mathscr{C}$ of locally constant functions, that is functions which are constant on each boundary component. On $\mathscr{C}$ and so also on $\mathscr{T}$ it is easy to check that the restriction of $\sigma$ is identically one.

Definition 3.2. For any subgroup $\mathscr{D}$ of $\mathscr{G}$ we define

\section{Lemma 3.1.}

$$
(\mathscr{D} \sigma)=\{\xi \in \mathscr{G}: \sigma(\xi, \delta)=\sigma(\delta, \xi), \delta \in \mathscr{D}\}
$$

(i) The group $\mathscr{C} \sigma$ is the connected component of the identity in $\mathscr{G}$, that is the subgroup of functions of the form $e^{i f}$ with $f$ in the Lie algebra.

(ii) The subgroup $\mathscr{T} \sigma$ consists of those $e^{i f}$ with $\sum_{j} \Delta_{j} f=0$.

(iii) The group $\mathscr{G}$ is the product of $\mathscr{C}$ with the vector subgroup $\mathscr{M}$ of functions in $\mathscr{C} \sigma$ having zero means on each boundary circle and a discrete group $\mathscr{Z} \cong \mathbf{Z}^{p+1}$ generated by elements having winding number 1 on one boundary circle and 0 on the rest. Thus $\mathscr{G}=\mathscr{Z} \times \mathscr{M} \times \mathscr{C}$ and $\mathscr{C} \sigma=\mathscr{M} \times \mathscr{C}$.

Proof. This follows immediately from the definitions (it is useful in this regard to consider $\mathscr{G}$ as the product of functions on the individual boundary components).

Proposition 3.2. The representation $\Gamma$ of $\mathscr{G}$ is induced in the sense of Mackey from the subgroup $\mathscr{C} \sigma$. 
Proof. The first step is to apply the Mackey analysis [M1] to the normal subgroup $\mathscr{C}$. We begin by choosing a 1 -dimensional $\sigma$-representation $\mu$ of $\mathscr{C}$, which, since $\sigma \equiv 1$, is an ordinary character. (The subgroup $\mathscr{C}$ is locally compact and satisfies the conditions needed to apply Mackey's techniques, so the fact that $\mathscr{G}$ is not locally compact is no obstacle.) The group $\mathscr{G}$ acts by sending $\mu$ to

$$
(\xi \cdot \mu)=\frac{\sigma(\xi, \cdot)}{\sigma(\cdot, \xi)} \mu
$$

It follows immediately that the stabiliser of $\mu$ is the subgroup $\mathscr{C} \sigma$. For a single boundary component the repeated action of elements of winding number 1 or -1 will send any character of a constant function to any other, and so $\mathscr{Z}$ acts transitively on the characters of $\mathscr{C}$. Mackey's Theorem tells us that we may as well take $\mu=1$, and that the $\sigma$-representation $\Gamma$ can be induced from a representation of $\mathscr{C} \sigma$ which is lifted from a $\sigma$-representation $\Phi$ of $(\mathscr{C} \sigma) / \mathscr{C} \cong \mathscr{M}$ completing the proof.

The next task is to determine the representation which is used to induce $\Gamma$. We begin by concentrating on $(\mathscr{C} \sigma) / \mathscr{C}$. There is a natural map $j$ from the subgroup $\mathscr{C} \sigma$ to the 1 -forms on $\partial \Sigma_{+}$, given by

$$
j(\xi)=\frac{1}{2 \pi i} d \log \xi=\frac{d \xi}{2 \pi i \xi} .
$$

The integral of $j(\xi)$ round each boundary component, which is the winding number of $\xi$, vanishes, but otherwise the only constraint is that $j(\xi)$ must be real on the boundary. The kernel of $j$ consists of the locally constant functions so that there is an induced isomorphism, which we shall also write as $j$ from $(\mathscr{C} \sigma) / \mathscr{C}$ to 1 -forms, satisfying the two constraints given above.

As with $\frac{1}{2}$-forms, the complexified space of 1 -forms on the boundary is the inductive limit of spaces of 1-forms on small collars surrounding $\partial \Sigma_{+}$, but for ease of notation we shall suppress those limits and just regard the boundary as the intersection of the Riemann surface $\Sigma_{+}$and its Schottky reflection $\Sigma_{-}$, whose union is the whole surface $\Sigma$. The Mayer-Vietoris sequence for forms gives in this case

$$
\begin{aligned}
0 & \rightarrow \Gamma(\Sigma, \mathcal{O}(K)) \rightarrow \Gamma\left(\Sigma_{+}, \mathcal{O}(K)\right) \oplus \Gamma\left(\Sigma_{-}, \mathcal{O}(K)\right) \rightarrow \Gamma\left(\partial \Sigma_{+}, \mathcal{O}(K)\right) \\
& \rightarrow H^{1}(\Sigma, \mathcal{O}(K)) \rightarrow 0 .
\end{aligned}
$$

The space $H^{1}(\Sigma, \mathcal{O}(K))$ is known to be one-dimensional, but forms on the boundary with vanishing winding numbers map to 0 , so that for these forms denoted by the suffix 0 the Mayer-Vietoris sequence reduces to

$$
0 \rightarrow \Gamma_{0}(\Sigma, \mathcal{O}(K)) \rightarrow \Gamma_{0}\left(\Sigma_{+}, \mathcal{O}(K)\right) \oplus \Gamma_{0}\left(\Sigma_{-}, \mathcal{O}(K)\right) \rightarrow \Gamma_{0}\left(\partial \Sigma_{+}, \mathcal{O}(K)\right) \rightarrow 0 .
$$

We therefore have an isomorphism

$$
\Gamma_{0}\left(\partial \Sigma_{+}, \mathcal{O}(K)\right) \cong \Gamma_{0}\left(\Sigma_{+}, \mathcal{O}(K)\right) \oplus \Gamma_{0}\left(\Sigma_{-}, \mathcal{O}(K)\right) / \Gamma_{0}(\Sigma, \mathcal{O}(K)) .
$$

In order to link in with standard Riemann surface theory we shall choose a basis of $A$ and $B$ cycles on our surface $\Sigma$ of genus $g$. This may be done along the lines indicated in [F]. For a start, we pick one of the boundary circles $A_{0}$ as a base circle, and take the remaining boundary circles $A_{1}^{0}, \ldots, A_{p}^{0}$ as $A$ cycles, (henceforth called $A^{0}$-cycles). For corresponding $B$ cycles we choose closed curves $B_{j}^{0}$ intersecting 
$A_{0}$ and $A_{j}^{0}$ just once and invariant (up to orientation reversal) under the Schottky involution. In $\Sigma_{+}$we choose further $A$ and $B$ cycles (called $A^{+}$and $B^{+}$cycles), $A_{1}^{+}, \ldots, A_{n}^{+}$and $B_{1}^{+}, \ldots, B_{n}^{+}$, with $n=(g-p) / 2$, and then complete the set with their images $A_{k}^{-}=A_{k}^{+}$and $B_{k}^{-}=\tilde{B}_{k}^{+}$under the Schottky involution. Here $n$ is the genus of the surface obtained by capping the boundary circles of $\Sigma_{+}$with discs. The space $\Gamma(\Sigma, \mathcal{O}(K))$ is $g$-dimensional and a basis $\omega_{1}^{0}, \ldots, \omega_{p}^{0}, \omega_{1}^{+}, \ldots, \omega_{n}^{+}, \omega_{1}^{-}, \ldots, \omega_{n}^{-}$may be chosen dual to $A$-cycles under the natural pairing given by integration round a cycle. More precisely, we take

$$
\int_{A_{k}^{s}} \omega_{l}^{t}=2 \pi i \delta_{s t} \delta_{k l},
$$

where $s$ and $t$ can be $0,+$ or - and $k$ and $l$ take appropriate integer values. The Schottky symmetries of the cycles and the fact that $\sim$ reverses orientation mean that $\omega_{k}^{-}=-\tilde{\omega}_{k}^{+}$, where $\tilde{\omega}$ denotes the complex conjugate of the image of the form $\omega$ under the Schottky involution. The integrals round $B$ cycles then give the period matrix:

$$
\int_{B_{k}^{s}} \omega_{l}^{t}=\tau_{k l}^{s t} .
$$

It follows from the behaviour of cycles and forms under the Schottky involution that the matrices $\tau^{++}$and $\tau^{--}$are conjugates, and so are $\tau^{+-}$and $\tau^{-+}$. Since the symmetry of the period matrix already means that $\tau^{+-}$is the transpose of $\tau^{-+}$, we deduce that both these matrices are self-adjoint.

By cutting the half space $\Sigma^{+}$along the $A^{+}$and $B^{+}$cycles it is possible to obtain a neat form for the infinitesimal 2-cocycle for functions whose differentials are holomorphic in $\Sigma^{+}$. In fact, writing $\Sigma_{+}^{\prime}$ for the cut surface, and arguing as in the derivation of the Riemann abelian relations $($ see $[\mathrm{H}])$ we obtain

$$
\begin{aligned}
s\left(f_{1}, f_{2}\right) & =\int_{\partial \Sigma_{+}} f_{2} d f_{1}+\sum f_{2}\left(c_{k}\right) \Delta_{k}\left(f_{1}\right) \\
& =\int_{\partial \Sigma_{+}^{\prime}} f_{2} d f_{1}-\sum_{k=1}^{n}\left(\int_{A_{k}^{+}} d f_{2} \int_{B_{k}^{+}} d f_{1}-\int_{A_{k}^{+}} d f_{1} \int_{B_{k}^{+}} d f_{2}\right)+\sum f_{2}\left(c_{k}\right) \Delta_{k}\left(f_{1}\right) .
\end{aligned}
$$

For holomorphic forms the first integral vanishes and for functions with winding number 0 on the original boundary the final sum vanishes too, leaving

$$
s\left(f_{1}, f_{2}\right)=\sum_{k=1}^{n}\left(\int_{A_{k}^{+}} d f_{1} \int_{B_{k}^{+}} d f_{2}-\int_{A_{k}^{+}} d f_{2} \int_{B_{k}^{+}} d f_{1}\right),
$$

which, using the fact that $j(\exp (i f))=d f / 2 \pi$, can also be expressed as

$$
s\left(f_{1}, f_{2}\right)=4 \pi^{2} \sum_{k=1}^{n}\left(\int_{A_{k}^{+}} j\left(e^{i f_{1}}\right) \int_{B_{k}^{+}} j\left(e^{i f_{2}}\right)-\int_{A_{k}^{+}} j\left(e^{i f_{2}}\right) \int_{B_{k}^{+}} j\left(e^{i f_{1}}\right)\right) .
$$

There is a similar expression for forms holomorphic on $\Sigma_{-}$.

Lemma 3.3. The space $\Gamma_{0}\left(\partial \Sigma_{+}, \mathcal{O}(K)\right)$ decomposes into a direct sum of the globally holomorphic forms $\Gamma_{0}(\Sigma, \mathcal{O}(K))$ with the forms holomorphic and exact on $\Sigma_{+}$and $\Sigma_{-}$respectively. 
Proof. Writing $\Gamma_{e}\left(\Sigma_{ \pm}, \mathcal{O}(K)\right)$ for the exact holomorphic forms on $\Sigma_{ \pm}$, we have

$$
\Gamma\left(\Sigma_{ \pm}, \mathcal{O}(K)\right) / \Gamma_{e}\left(\Sigma_{ \pm}, \mathcal{O}(K)\right) \cong H^{1}\left(\Sigma_{ \pm}\right),
$$

so that the quotient has dimension $n+p$, and its subspace $\Gamma_{0}\left(\Sigma_{ \pm}, \mathcal{O}(K)\right)$ / $\Gamma_{e}\left(\Sigma_{ \pm}, \mathcal{O}(K)\right)$ has dimension $n$. This means that

$$
\Gamma_{0}\left(\Sigma_{+}, \mathcal{O}(K)\right) / \Gamma_{e}\left(\Sigma_{+}, \mathcal{O}(K)\right) \oplus \Gamma_{0}\left(\Sigma_{-}, \mathcal{O}(K)\right) / \Gamma_{e}\left(\Sigma_{-}, \mathcal{O}(K)\right)
$$

has dimension $2 n=\operatorname{dim}\left(\Gamma_{0}(\Sigma, \mathcal{O}(K))\right)$.

On the other hand $\Gamma(\Sigma, \mathcal{O}(K))$ intersects each of $\Gamma_{e}\left(\Sigma_{ \pm}, \mathcal{O}(K)\right)$ trivially, as can be seen by the following argument. If $\alpha \in \Gamma(\Sigma, \mathcal{O}(K))$ is equal to $d \phi$ on $\Sigma_{\mp}$, then $\tilde{\alpha}=d \bar{\phi}$ on $\Sigma_{ \pm}$and

$$
\int_{\Sigma_{ \pm}} d \phi \wedge d \bar{\phi}=\int_{\Sigma_{ \pm}} d \phi \wedge \tilde{\alpha}=\int_{\partial \Sigma_{ \pm}} \phi \tilde{\alpha}
$$

This vanishes by Cauchy's theorem, so that $d \phi=0$. We may therefore form the direct sum

$$
\Gamma_{e}\left(\Sigma_{+}, \mathcal{O}(K)\right) \oplus \Gamma_{e}\left(\Sigma_{-}, \mathcal{O}(K)\right) \oplus \Gamma_{0}(\Sigma, \mathcal{O}(K)),
$$

which is a subspace of $\Gamma_{0}\left(\Sigma_{+}, \mathcal{O}(K)\right) \oplus \Gamma_{0}\left(\Sigma_{-}, \mathcal{O}(K)\right)$. Since, by the previous observation, its codimension is 0 , we deduce that

$$
\Gamma_{0}\left(\Sigma_{+}, \mathcal{O}(K)\right) \oplus \Gamma_{0}\left(\Sigma_{-}, \mathcal{O}(K)\right)=\Gamma_{e}\left(\Sigma_{+}, \mathcal{O}(K)\right) \oplus \Gamma_{e}\left(\Sigma_{-}, \mathcal{O}(K)\right) \oplus \Gamma_{0}(\Sigma, \mathcal{O}(K)) .
$$

We may therefore rewrite

$$
\Gamma_{0}\left(\partial \Sigma_{+}, \mathcal{O}(K)\right) \cong \Gamma_{0}\left(\Sigma_{+}, \mathcal{O}(K)\right) \oplus \Gamma_{0}\left(\Sigma_{-}, \mathcal{O}(K)\right) / \Gamma_{0}(\Sigma, \mathcal{O}(K))
$$

as

$$
\Gamma_{e}\left(\Sigma_{+}, \mathcal{O}(K)\right) \oplus \Gamma_{e}\left(\Sigma_{-}, \mathcal{O}(K)\right) \oplus \Gamma_{0}(\Sigma, \mathcal{O}(K))
$$

completing the proof.

Lemma 3.3 also enables us to factorise the vector group $\mathscr{M}$. We introduce the two subgroups

$$
\mathscr{W}=\left\{m \in \mathscr{M}: j(m)=\left.\xi\right|_{\partial \Sigma_{+}}, \xi \in \Gamma_{0}(\Sigma, \mathcal{O}(K))\right\},
$$

and

$$
\mathscr{V}=\left\{m \in \mathscr{M}: j(m)=\left.\xi_{+}\right|_{\partial \Sigma_{+}}+\left.\xi_{-}\right|_{\partial \Sigma_{-}}, \xi_{ \pm} \in \Gamma_{e}\left(\Sigma_{ \pm}, \mathcal{O}(K)\right)\right\} .
$$

Then by Lemma $3.3, \mathscr{M}=\mathscr{V} \times \mathscr{W}$.

Lemma 3.4. Let $\hat{\Sigma}_{+}$denote a closed surface obtained by capping $\Sigma_{+}$. Then

$$
\mathscr{W} \cong H^{1}\left(\hat{\Sigma}_{+}, \mathbf{R}\right)
$$

and $s \mid \mathscr{W} \times \mathscr{W}$ corresponds to the canonical symplectic form on $H^{1}$.

Proof. The forms which represent elements of $\mathscr{W}$ are real on the boundary and can therefore be represented by real harmonic forms rather than complex holomorphic forms. The real dimension of $\mathscr{W}$ is the same as the complex dimension of $\Gamma_{0}(\Sigma, \mathcal{O}(K))$, that is $2 n$. The cohomology of $\hat{\Sigma}_{+}$is represented by harmonic forms and these are determined by their restrictions to $\Sigma_{+}$(since they can be reconstructed 
on the caps from the boundary data). From the decomposition of the preceding lemma it follows that these forms determine the same cohomology classes as the restrictions to $\Sigma_{+}$of "real" forms in $\Gamma_{0}(\Sigma, \mathcal{O}(K))$. Since $\operatorname{dim} \mathscr{W}=\operatorname{dim} H^{1}\left(\hat{\Sigma}_{+}, \mathbf{R}\right)$, the isomorphism now follows. Equation (3.6) and its predecessor show that $s \mid \mathscr{W} \times \mathscr{W}$ is the canonical symplectic form on $H^{1}\left(\hat{\Sigma}_{+}, \mathbf{R}\right)$.

Corollary 3.5. The matrix $\tau^{+-}$is non-singular.

Proof. The 2-cocycle is equivalent to a symplectic form, and therefore nondegenerate. On the other hand Eq. (3.6) shows that if $\omega_{k}^{s}=d f_{k}^{s} / 2 \pi i$, then we have

$$
s\left(f_{l}^{+}, f_{m}^{+}\right)=4 \pi^{2} \sum_{k=1}^{n}\left(2 \pi i \delta_{k l} \tau_{k m}^{++}-2 \pi i \delta_{k m} \tau_{k l}^{++}\right)=8 \pi^{3} i\left(\tau_{l m}^{++}-\tau_{m l}^{++}\right)=0,
$$

by symmetry of $\tau$, and

$$
s\left(f_{l}^{-}, f_{m}^{+}\right)=4 \pi^{2} \sum_{k=1}^{n}\left(0-2 \pi i \delta_{k m} \tau_{k l}^{+-}\right)=8 \pi^{3} i \tau_{l m}^{+-} .
$$

The non-degeneracy of $s$ now gives the result.

It is now possible to give a more explicit form of the decomposition in Lemma 3.3 , since for any holomorphic form $\xi \in \Gamma_{0}\left(\Sigma_{-}, \mathcal{O}(K)\right)$ the form

$$
\xi_{0}=\xi-\sum_{k=1}^{n} \omega_{k}^{+} \int_{A_{k}^{-}} \xi
$$

has vanishing integrals round all the $A^{-}$-cycles, and

$$
\xi_{e}=\xi_{0}-\sum_{k, l=1}^{n} \omega_{k}^{-}\left(\tau^{-+}\right)_{k l}^{-1} \int_{B_{l}^{-}} \xi_{0}
$$

has vanishing integrals round all cycles in the half space $\Sigma_{-}$. Its integral is a well defined holomorphic function whose differential is $\xi_{e}$. This shows how to decompose $\xi$ as the sum of an exact form and a linear combination of globally holomorphic forms.

Since the group $\mathscr{W}$ is finite-dimensional and the 2-cocycle is non-degenerate, the Stone-von Neumann uniqueness theorem ensures that it has a unique irreducible $\sigma$-representation. The obvious way to construct the desired representation $\Phi$ of

$$
(\mathscr{C} \sigma) / \mathscr{C} \cong \mathscr{M} \cong \mathscr{V} \times \mathscr{W}
$$

is therefore to take the tensor product of the unique irreducible representation $\Upsilon$ of $\mathscr{W}$ with the Fock representation $\Pi$ of $\mathscr{V}$ whose cyclic vector is annihilated by $\Gamma_{e}\left(\Sigma_{-}, \mathcal{O}(K)\right)$.

To see that this is the correct representation we note that locally in a neighbourhood of any boundary circle apart from the finite-dimensional space $\Gamma_{0}(\Sigma, \mathcal{O}(K))$ the decomposition into $\Gamma_{e}\left(\Sigma_{+}, \mathcal{O}(K)\right) \oplus \Gamma_{e}\left(\Sigma_{-}, \mathcal{O}(K)\right)$ agrees with the usual Hardy space decomposition and so defines an equivalent Fock representation. This establishes the main result of this section.

Proposition 3.6. The $\sigma$-representation $\Gamma$ of $\mathscr{G}$ is equivalent to an induced representation

$$
\Gamma \equiv \sigma-\operatorname{ind}_{\mathscr{C} \sigma}^{\mathscr{G}} \Upsilon^{\prime} \otimes \Pi^{\prime}
$$


where $\Upsilon^{\prime}$ and $\Pi^{\prime}$ are the lifts to $\mathscr{C} \sigma$ of Fock representations of $\mathscr{W}$ and $\mathscr{V}$ respectively.

An alternative form for this representation follows from the fact that $\mathscr{G}=\mathscr{V} \times \mathscr{Z} \times \mathscr{W} \times \mathscr{C}$. Extending the Fock representation $\Pi$ trivially from $\mathscr{V}$ to $\mathscr{G}$, the permanence relation tells us that as a representation of $\mathscr{V} \times(\mathscr{Z} \times \mathscr{W} \times \mathscr{C})$,

$$
\Gamma \equiv \Pi \otimes \sigma-\operatorname{ind}_{\mathscr{W} \times \mathscr{C}}^{\mathscr{Z} \times \mathscr{C}} \Upsilon
$$

\section{The Fermion Vacuum}

Having found a convenient realisation of the representation of the bosonic group $\mathscr{G}$, the next task is to identify the fermion cyclic vector $\Omega$ within this representation.

Lemma 4.1. The vector $\Omega$ may be identified with the tensor product of the Fock vacuum $\Omega_{\Pi}$ for $\mathscr{V}$ with a vector $\Omega_{\Upsilon}$ in the representation space for $\mathscr{Z} \times \mathscr{W} \times \mathscr{C}$.

Proof. Notice first that if multiplication by $\xi \in \mathscr{G}$ maps $\mathscr{K}_{-}=\Gamma\left(\Sigma_{-}, \mathcal{O}\left(K^{\frac{1}{2}}\right)\right)$ into itself, then for all $\alpha \in \mathscr{K}_{-}$we have

$$
\Psi(\alpha) \Gamma(\xi)^{-1} \Omega=\Gamma(\xi)^{-1} \Psi(\xi \cdot \alpha) \Omega=0 .
$$

Since $\Omega$ is uniquely defined, up to multiples, as the vector annihilated by $\Psi\left(\mathscr{K}_{-}\right)$ it follows that $\Gamma(\xi)^{-1} \Omega$ is a multiple of $\Omega$ or, equivalently, that for suitable $\chi$

$$
\Gamma(\xi) \Omega=\chi(\xi) \Omega .
$$

We could equally well replace $\Gamma(\xi)$ by $\chi(\xi)^{-1} \Gamma(\xi)$ as the implementor of the loop group automorphisms of the fermion algebra, and then $\Omega$ is fixed by $\Gamma(\xi)$.

The most obvious example of such $\xi$ is afforded by the globally constant functions, $\mathscr{T}$, so we may assume that these fix $\Omega$. Since for any $\eta \in \mathscr{G}$ we have

$$
\Gamma(\xi) \Gamma(\eta) \Omega=\frac{\sigma(\eta, \xi)}{\sigma(\xi, \eta)} \Gamma(\eta) \Gamma(\xi) \Omega=\frac{\sigma(\eta, \xi)}{\sigma(\xi, \eta)} \Gamma(\eta) \Omega,
$$

we see that for any $\eta \in \mathscr{G}$ the vector $\Gamma(\eta) \Omega$ is also an eigenvector, but that the eigenvalue is only 1 when $\eta \in(\mathscr{T} \sigma)$. The consequent orthogonality condition shows that the generating function $\langle\Omega, \Gamma(\eta) \Omega\rangle$ must vanish unless $\eta \in(\mathscr{T} \sigma)$. The fact that $\Omega$ is fixed by $\Gamma(\mathscr{T})$ means that there the generating function is lifted from a function on the quotient group $(\mathscr{T} \sigma) / \mathscr{T}$.

Similarly, if we let $f$ be an element of the Lie algebra of $\mathscr{G}$ which maps $\mathscr{K}_{-}$ into itself, then with $d \Gamma$ the Lie algebra representation obtained from $\Gamma$ we have

$$
\Psi(\alpha) d \Gamma((f) \Omega=d \Gamma(f) \Psi(\alpha) \Omega-\Psi(f \cdot \alpha) \Omega=0
$$

for all $\alpha \in \mathscr{K}_{-}$, and so $d \Gamma(f) \Omega$ is a multiple of $\Omega$. Again, by modifying the implementors by a scalar we may as well assume that $d \Gamma(f) \Omega=0$.

The same arguments may be applied for $f$ in the complex Lie algebra. By results of [CP] the vector $\Omega$ is in the domain of the elements $\xi$ in the complexification of $\mathscr{G}$, and hence the same arguments apply to them as well. The obvious elements in the Lie algebra which fix $\mathscr{K}_{-}$are those in $\Gamma_{e}\left(\Sigma_{-}, \mathcal{O}(K)\right)$, and we accordingly deduce that the fermion cyclic vector is killed by these. This completes the proof. 
The main problem now is to analyse the representation of the subgroup $\mathscr{Z} \times \mathscr{W} \times \mathscr{C}$. This task is considerably simplified by noting that the elements of $\mathscr{Z} \times \mathscr{C}$ can also be related to forms. For a start, it is clear that for a general function $\phi$ in $\mathscr{G}$ the winding numbers on the boundary are given by the integrals

$$
\frac{1}{2 \pi i} \int_{A_{r}^{0}} \frac{d \phi}{\phi}=\int_{A_{r}^{0}} j(\phi),
$$

where the map $j$ of Eq. (3.5) has been extended in the obvious way. If $\phi$ is in the subgroup $\mathscr{T} \sigma$, so that the total winding number vanishes, then the form

$$
j(\phi)-\sum_{r=1}^{p} \omega_{r}^{0} \int_{A_{r}^{0}} j(\phi)
$$

has vanishing periods round all the boundary cycles, and so it is of the form $j\left(\phi_{0}\right)$ for $\phi_{0} \in \mathscr{C} \sigma$. In general, the periods of $j(\phi)$ round the boundary cycles must all necessarily be integers.

We have so far ignored one rather subtle but important side effect of using these forms. The values of the function associated with a form $\eta$ differ at the points where $B_{j}^{0}$ meets $A_{0}$ and $A_{r}^{0}$ by the exponential of

$$
2 \pi i \underset{B_{r}^{0} \cap \Sigma_{-}}{\int} \eta
$$

(We can simplify some of the expressions by choosing the cycle $B_{j}^{0}$ so that it intersects $A_{j}^{0}$ in $c_{j}$ ). The $\mathscr{T}$-coset of a locally constant function is uniquely determined by the differences between its values on $A_{0}$ and the other $A^{0}$ cycles, and one side effect of using the forms $\omega_{1}^{0}, \ldots, \omega_{p}^{0}$ to encode information about $\mathscr{Z}$, is that the corresponding functions have non-trivial components in $\mathscr{C} / \mathscr{T}$-cosets. (This problem did not arise with the other forms because there was a natural decomposition splitting them off from the locally constant functions.)

Since the boundary is invariant under the Schottky involution, the complex conjugate of the period of the form $\eta$ round $A_{j}^{0}$ is

$$
\frac{-1}{2 \pi i} \int_{A_{j}^{0}} \bar{\eta}=\frac{-1}{2 \pi i} \int_{A_{j}^{0}} \tilde{\eta} .
$$

If $\tilde{\eta}=-\eta$ then this period will automatically be real, and this is the case for each of the forms $\omega_{1}^{0}, \ldots, \omega_{p}^{0}$. Now consider, for such a form $\eta$ the real part of the difference between the values of the associated function on boundary components, that is

$$
\frac{1}{2}\left(\int_{B_{r}^{0} \cap \Sigma_{-}} \eta+\int_{B_{r}^{0} \cap \Sigma_{-}} \bar{\eta}\right) .
$$

Recalling the way the Schottky involution acts on forms and that it reverses orientation, this may be rewritten as

$$
\frac{1}{2}\left(\int_{B_{r}^{0} \cap \Sigma_{-}} \eta-\int_{B_{r}^{0} \cap \Sigma_{+}} \tilde{\eta}\right),
$$


which reduces to

$$
\frac{1}{2}\left(\underset{B_{r}^{0} \cap \Sigma_{-}}{\int} \eta+\underset{B_{r}^{0} \cap \Sigma_{+}}{\int} \eta\right)=\frac{1}{2} \int_{B_{r}^{0}} \eta
$$

For the particular forms $\omega_{k}^{0}$ these $B^{0}$ integrals are linked to those round the $A^{0}$ cycles by the period matrix. Since the $A^{0}$ periods have to be integral, there are also integrality constraints on the $B^{0}$ periods. In this way $\Gamma(\Sigma, \mathcal{O}(K))$ can be regarded as the direct sum of $\Gamma_{0}(\Sigma, \mathcal{O}(K))$ with part of the complexified Lie algebra of $\mathscr{Z} \times \mathscr{C}$.

Lemma 4.2. There is a complex lattice subgroup $\mathscr{L}$ in $\Gamma(\Sigma, \mathcal{O}(K))$ which has the vector $\Omega_{\Upsilon}$ as an eigenvector.

Proof. First we note that the complexification of the finite-dimensional vector group $\mathscr{W}$ can be identified with $\Gamma_{0}\left(\Sigma_{+}, \mathcal{O}(K)\right)$ using the map $j$ in (3.5). Within $\Gamma_{0}(\Sigma, \mathcal{O}(K))$ the forms whose integrals round all $A^{-}$and $B^{-}$cycles are integers can be integrated to give well-defined holomorphic functions on $\Sigma_{-}$. The existence of a lattice $\mathscr{L}_{0}$ with this property can be shown by an argument similar to that splitting a form into exact and globally holomorphic parts, and it is spanned by

$$
\alpha_{k}=\sum_{l=1}^{n}\left(\tau^{-+}\right)_{l k}^{-1} \omega_{l}^{+}
$$

and

$$
\beta_{k}=(2 \pi i)^{-1}\left(\omega_{k}^{-}-\sum_{l, m=1}^{n} \tau_{l k}^{--}\left(\tau^{-+}\right)_{m l}^{-1} \omega_{m}^{+}\right)
$$

for $k=1, \ldots, n$. These preserve $\mathscr{K}_{-}$and since the group is only finite dimensional it can be complexified and general theory asserts that $\Omega$ lies in its domain, so that $\Omega_{\Upsilon}$ is an eigenvector for $\mathscr{L}_{0}$, and can be assumed fixed by that lattice.

We can, however, expand $\mathscr{L}_{0}$ to a larger lattice. The forms

$$
\gamma_{k}=(2 \pi i)^{-1}\left(\omega_{k}^{0}-\sum_{l, m=1}^{n} \tau_{l k}^{-0}\left(\tau^{-+}\right)_{m l}^{-1} \omega_{m}^{+}\right)
$$

also have integer periods round all cycles in $\Sigma_{-}$including the $A^{0}$ cycles on the boundary. Each therefore has boundary values of the form $j(\xi)$ for some element $\xi$ of the complexification of $\mathscr{G}$ which preserves $\mathscr{K}_{-}$. The same applies for integral linear combinations of such elements,so that this lattice should be joined to $\mathscr{L}_{0}$ to generate a larger lattice $\mathscr{L}$ which sits inside $\Gamma(\Sigma, \mathcal{O}(K))$. By the above arguments $\Omega_{\Upsilon}$ is certainly an eigenvector of the complex lattice $\mathscr{L}$.

Remarks 4.1. We recall that a subgroup is said to be isotropic if the restriction of the 2-cocycle $\sigma$ is trivial, or, equivalently, in a form which extends to the complexification, if the restriction of $s$ takes only integer multiples of $4 \pi^{2}$. An isotropic lattice is said to be maximal if it is not a proper subgroup of a larger isotropic subgroup. The sublattice $\mathscr{L}_{0}$ is maximal isotropic in this sense, which can be seen from the fact that the other variant of (3.6) gives

$$
s\left(f_{1}, f_{2}\right)=-4 \pi^{2} \sum_{k=1}^{n}\left(\int_{A_{k}^{-}} j\left(e^{i f_{1}}\right) \int_{B_{k}^{-}} j\left(e^{i f_{2}}\right)-\int_{A_{k}^{-}} j\left(e^{i f_{2}}\right) \int_{B_{k}^{-}} j\left(e^{i f_{1}}\right)\right)
$$


and the forms have been chosen to have only negative periods round the $A^{-}$and $B^{-}$cycles.

To summarise our findings to date we have shown that the fermion cyclic vector is the tensor product of the Fock cyclic vector $\Omega_{\Pi}$ annihilated by $\Gamma_{e}\left(\Sigma_{-}, \mathcal{O}(K)\right)$, and an eigenvector, $\Omega_{\Upsilon}$, of the complex lattice $\mathscr{L}$. Moreover, since we know the generators of $\mathscr{L}$ we can easily find an explicit formula for $\Omega_{\Upsilon}$. Now real isotropic lattices do not have eigenvectors, but only generalised eigenvectors in some suitably rigged version of the Hilbert space, though these are uniquely determined when the lattice is maximal. However, in the complex case, there are genuine eigenvectors which are still unique, as we shall now prove.

The vector $\Omega_{\Upsilon}$ is in the space of the representation induced from $\Upsilon$ on $\mathscr{W} \times \mathscr{C}$, or equivalently the tensor product of the $\sigma$-representations $\Upsilon$ on $\mathscr{W}$ and

$$
\Xi=\sigma-\operatorname{ind}_{\mathscr{C}}^{\mathscr{Z} \times \mathscr{C}} \Upsilon
$$

of $\mathscr{Z} \times \mathscr{C}$. The representation space for $\Xi$ is $\ell^{2}(\mathscr{Z})$, but we already know that $\Omega_{\Upsilon}$ is supported on $\mathscr{C} \sigma$, so we may as well work in the subspace $\ell^{2}(\mathscr{C} \sigma) \cong \ell^{2}\left(\mathbf{Z}^{p}\right)$. Let $\phi$ be a function in $\mathscr{C} \sigma \times \mathscr{C}$ whose winding number round $A_{k}^{0}$ is $m_{k}$ and with locally constant part $\exp \left(i \mu_{k}\right)$ on that circle and 1 on $A_{0}$. Then for any $\psi$ in the representation space and $l \in \mathbf{Z}^{p}$, we have

$$
(\Xi(\phi) \psi)(l)=e^{i \frac{\pi}{4} m \cdot m} e^{i l \cdot \mu} \psi(l-m) .
$$

In order to obtain $\Upsilon$ in a convenient form let us first note that

$$
\mathscr{W}_{\mathbf{C}}=\operatorname{span}\left\{\alpha_{k}\right\} \oplus \operatorname{span}\left\{\tilde{\alpha_{k}}\right\}
$$

provides a polarisation of the complexification of $\mathscr{W}$. We can therefore realise $\Upsilon$ on the Fock-Bargmann Segal space of holomorphic functions on $\operatorname{span}\left\{\alpha_{k}\right\}$. For $\Psi$ such a function, $\phi \in \mathscr{W}$ satisfying $j(\phi)=\sum x_{k} \alpha_{k}+\tilde{x}_{k} \tilde{\alpha}_{k}$, and $z=\sum z_{k} \alpha_{k}$, we have

$$
(\Upsilon(\phi) \Psi)(z)=e^{-2 \pi^{2} \tilde{x}\left(\tau^{-+}\right)^{-1} x} e^{4 \pi^{2} \tilde{x}\left(\tau^{-+}\right)^{-1} z} \Psi(z-x) .
$$

(We may readily check that this gives the correct 2-cocycle, as determined by our earlier calculation of $s$ for functions associated to holomorphic forms.) The inner product is given by

$$
\left.\left\langle\Psi_{1}, \Psi_{2}\right\rangle=\int \overline{\Psi_{1}(z)} \exp \left(-2 \pi^{2} \tilde{z}\left(\tau^{-+}\right)^{-1} z\right)\right) \Psi_{2}(z) d z d \bar{z} .
$$

As a vector in the tensor product space $\Omega_{\Upsilon}$ is a holomorphic function of $z$ and a square-summable function of $l$. Since it is an eigenvector of each of the lattice elements $\alpha_{k}$ we take integral $x_{k}$ and vanishing $\tilde{x}_{k}$ to deduce that $\Omega_{\Upsilon}(z-x, l)$ is a multiple of $\Omega_{\Upsilon}$. Taking the eigenvalue to be 1 in accordance with our previous discussion, $\Omega_{\Upsilon}$ is periodic in $z$ and of the form

$$
\Omega_{\Upsilon}(z)=\sum_{n \in \mathbf{Z}^{p}} e^{2 \pi i n \cdot z} \Omega(n, l) .
$$

To consider the effect of the other lattice generators $\beta_{k}$, we first note that

$$
\tilde{\alpha}_{k}=-\sum_{l}\left(\tau^{+-}\right)_{l k}^{-1} \omega_{l}^{-},
$$

so that

$$
\beta_{k}=\frac{-1}{2 \pi i} \sum_{l}\left(\tau_{l k}^{+-} \tilde{\alpha}_{l}+\tau_{l k}^{--} \alpha_{l}\right) .
$$


Thus for integral $m_{k}, \sum_{k} m_{k} \beta_{k}$ has components $\tilde{x}=-\tau^{+-} \cdot m / 2 \pi i$ and $x=$ $-\tau^{--} \cdot m / 2 \pi i$. The action of this lattice element on $\Omega_{\Upsilon}(z, l)$ produces

$$
\begin{aligned}
& e^{\frac{1}{2} m \cdot \tau^{--} \cdot m} e^{2 \pi i m \cdot z} \Omega_{\Upsilon}\left(z-\frac{i}{2 \pi} \tau^{--} \cdot m, l\right) \\
& =\sum_{n} e^{\frac{1}{2} m \cdot \tau^{--} \cdot m} e^{2 \pi i m \cdot z} e^{2 \pi i n \cdot z} e^{\frac{1}{2} n \cdot \tau^{--} \cdot m} \Omega(n, l) \\
& =\sum_{n} e^{\frac{1}{2}(m+n) \cdot \tau^{--} \cdot(m+n)} e^{-\frac{1}{2} n \cdot \tau^{--} \cdot n} e^{2 \pi i(m+n) \cdot z} \Omega(n, l) .
\end{aligned}
$$

If this is a multiple $\lambda(m)$ of $\Omega_{\Upsilon}$ then we must have

$$
e^{\frac{1}{2}(m+n) \cdot \tau^{--} \cdot(m+n)} e^{-\frac{1}{2} n \cdot \tau^{--} \cdot n} \Omega(n, l)=\lambda(m) \Omega(m+n, l) .
$$

Rearranging this as

$$
e^{-\frac{1}{2} n \cdot \tau^{--} \cdot n} \Omega(n, l)=\lambda(m) e^{-\frac{1}{2}(m+n) \cdot \tau^{--} \cdot(m+n)} \Omega(m+n, l),
$$

and setting $n=0$, we see that

$$
\Omega(m, l)=\lambda(m)^{-1} e^{\frac{1}{2} m \cdot \tau^{--} \cdot m} \Omega(0, l) .
$$

Substituting back into the functional equation we see that $\chi_{1}(m)=\lambda(m)^{-1}$ is a character.

Finally we must consider the action of the elements $\gamma_{k}$. Since

$$
\sum m_{k} \gamma_{k}=\sum \frac{m_{k}}{2 \pi i}\left(\omega_{k}^{0}-\tau_{l k}^{-0} \alpha_{l}\right)
$$

we see that when we take an element with winding numbers $m_{k}$, not only do we obtain a vector of local constants $\mu=-i \frac{1}{2} \tau^{00} m$, but also a vector $x=-\tau^{-0} \cdot m / 2 \pi i$ in $\mathscr{W}_{\mathbf{C}}$. The action of this element therefore sends $\Omega_{\Upsilon}(z, l)$ to

$$
\begin{aligned}
& e^{i \frac{\pi}{4} m \cdot m} e^{\frac{1}{2} l \cdot \tau^{00} \cdot m} \Omega_{\Upsilon}\left(z+\frac{1}{2 \pi i} \tau^{-0} \cdot m, l-m\right) \\
& =\sum_{n} e^{i \frac{\pi}{4} m \cdot m} e^{\frac{1}{2} l \cdot \tau^{00} \cdot m} e^{\frac{1}{2} n \cdot \tau^{-0} \cdot m} e^{\pi i n \cdot z} \Omega(n, l-m) .
\end{aligned}
$$

The condition that this be a multiple $\chi(m)$ of $\Omega_{\Upsilon}(z, l)$ is that

$$
e^{i \frac{\pi}{4} m \cdot m} e^{\frac{1}{2} l \cdot \tau^{00} \cdot m} e^{\frac{1}{2} n \cdot \tau^{-0} \cdot m} \Omega(n, l-m)=\chi(m) \Omega(n, l),
$$

or, equivalently, that

$$
\begin{aligned}
& e^{-\frac{1}{4}(l-m) \cdot \tau^{00} \cdot(l-m)} e^{-\frac{1}{2} n \cdot \tau^{-0} \cdot(l-m)} \Omega(n, l-m) \\
& \quad=\left(\chi(m) e^{-\frac{1}{4} m \cdot \tau^{00} \cdot m} e^{-i \frac{\pi}{4} m \cdot m}\right) e^{-\frac{1}{4} l \cdot \tau^{00} \cdot l} e^{-\frac{1}{2} n \cdot \tau^{-0} \cdot l} \Omega(n, l) .
\end{aligned}
$$

Setting $m=l$ we see that

$$
e^{-\frac{1}{4} l \cdot \tau^{00} \cdot l} e^{-\frac{1}{2} n \cdot \tau^{-0} \cdot l} \Omega(n, l)=\left(\chi(l) e^{-\frac{1}{4} l \cdot \tau^{00} \cdot l} e^{-i \frac{\pi}{4} l \cdot l}\right)^{-1} \Omega(n, 0),
$$


and on substituting back we see that $\chi_{2}(l)=\exp \left(\frac{1}{4} l \cdot\left(\tau^{00}+i \pi\right) \cdot l\right) \chi(l)^{-1}$ is a character.

Combining all these results we obtain the explicit formula

$$
\Omega(n, l)=\chi_{1}(n) \chi_{2}(l) \exp \left(\frac{1}{4} l \cdot \tau^{00} \cdot l+n \cdot \tau^{-0} \cdot l+\frac{1}{2} n \cdot \tau^{--} \cdot n\right) \Omega(0,0),
$$

confirming that $\Omega_{\Upsilon}$ is indeed determined up to multiples. As noted earlier, we may, without loss of generality, assume that $\chi_{1}$ and $\chi_{2}$ are trivial, so that

$$
\Omega(n, l)=\exp \left(\frac{1}{4} l \cdot \tau^{00} \cdot l+n \cdot \tau^{-0} \cdot l+\frac{1}{2} n \cdot \tau^{--} \cdot n\right) \Omega(0,0) .
$$

We can now obtain an explicit formula for the generating function for the representation. First we note that for elements of the real group the vector $\tilde{x}$ is just the complex conjugate of $x$, so taking a function $\phi$ associated with winding numbers $m_{k}$, local constants $\mu_{k}$ and vectors $x$ and $\tilde{x}=\bar{x}$, we have

$$
\begin{aligned}
( & \left.\Gamma(\phi) \Omega_{\Upsilon}\right)(z, l) \\
& =e^{-i \frac{\pi}{4} m \cdot m} e^{-2 \pi^{2} \bar{x}\left(\tau^{-+}\right)^{-1} x} e^{4 \pi^{2} \bar{x}\left(\tau^{-+}\right)^{-1} z} e^{i l \cdot \mu} \Omega_{\Upsilon}(z-x, l-m) \\
& =\sum_{n} e^{-i \frac{\pi}{4} m \cdot m} e^{-2 \pi^{2} \bar{x}\left(\tau^{-+}\right)^{-1} x} e^{4 \pi^{2} \bar{x}\left(\tau^{-+}\right)^{-1} z} e^{i l \cdot \mu} e^{2 \pi i n \cdot(z-x)} \Omega(n, l-m) \\
& =\sum_{n} e^{-i \frac{\pi}{4} m \cdot m} e^{-2 \pi^{2} \bar{x}\left(\tau^{-+}\right)^{-1} x} e^{-2 \pi i n \cdot x} e^{2 \pi i\left(n-2 \pi i\left(\tau^{+-}\right)^{-1} \bar{x}\right) \cdot z} e^{i l \cdot \mu} \Omega(n, l-m) .
\end{aligned}
$$

Now the inner product of the exponential functions $\exp (2 \pi i k \cdot z)$ and $\exp (2 \pi i n \cdot z)$ in Fock space is $\exp \left(k \cdot \tau^{+-} \cdot n\right)$, so we may write the generating function as

$$
\begin{aligned}
\left\langle\Omega_{\Upsilon}, \Gamma(\phi) \Omega_{\Upsilon}\right\rangle= & \sum_{n, k, l} e^{-i \frac{\pi}{4} m \cdot m} e^{k \cdot \tau^{+-} \cdot\left(n-2 \pi i\left(\tau^{+-}\right)^{-1} \bar{x}\right)} e^{-\pi^{2} \bar{x}\left(\tau^{-+}\right)^{-1} x} e^{-i \pi n \cdot x} \\
& \times e^{i l \cdot \mu} \overline{\Omega(k, l)} \Omega(n, l-m) \\
= & \sum_{n, k, l} e^{-i \frac{\pi}{4} m \cdot m} e^{-2 \pi^{2} \bar{x}\left(\tau^{-+}\right)^{-1} x} e^{-2 \pi i n \cdot x} e^{-2 \pi i k \cdot \bar{x}} e^{i l \cdot \mu} e^{k \cdot \tau^{+-} \cdot n} e^{-\frac{1}{2} l \cdot \tau^{00} \cdot m} \\
& \times e^{\frac{1}{4} m \cdot \tau^{00} \cdot m} e^{-n \cdot \tau^{-0} \cdot m} \overline{\Omega(k, l)} \Omega(n, l) .
\end{aligned}
$$

Now, bearing in mind the fact that conjugating blocks in $\tau$ simply interchanges + and - superscripts, the terms which do not involve the group elements are

$$
\begin{aligned}
e^{k \cdot \tau^{+-} \cdot n} \overline{\Omega(k, l)} \Omega(n, l)= & e^{\frac{1}{4}\left(l \cdot \tau^{00} \cdot l+4 k \cdot \tau^{+0} \cdot l+2 k \cdot \tau^{++} \cdot k\right)} e^{k \cdot \tau^{+-} \cdot n} \\
& \times e^{\frac{1}{4}\left(l \cdot \tau^{00} \cdot l+4 n \cdot \tau^{-0} \cdot l+2 n \cdot \tau^{--} \cdot n\right)}|\Omega(0,0)|^{2} .
\end{aligned}
$$

The exponentials can be rewritten in terms of the vector $q=(k, l, m)$, as $\exp \left(\frac{1}{2}(q \cdot \tau \cdot q)\right.$ ). (Since the period matrix $\tau$ has negative definite real part this will give a convergent series, confirming that $\Omega_{\Upsilon}$ is square-integrable, as general theory predicts.) In fact, dropping the constant factor $|\Omega(0,0)|^{2}$, the generating function 
now collapses to

$$
\begin{aligned}
\sum_{n, k, l} e^{i \frac{\pi}{4} m \cdot m} e^{-2 \pi^{2} \bar{x}\left(\tau^{-+}\right)^{-1} x} e^{-2 \pi i n \cdot x} e^{-2 \pi i k \cdot \bar{x}} e^{i l \cdot \mu} e^{-\frac{1}{2} l \cdot \tau^{00} \cdot m} \\
\quad \times e^{\frac{1}{4} m \cdot \tau^{00} \cdot m} e^{-\frac{1}{2} n \cdot \tau^{-0} \cdot m} e^{\frac{1}{2}(q \cdot \tau \cdot q)} \\
=e^{-\pi^{2} \bar{x}\left(\tau^{-+}\right)^{-1} x} e^{\frac{1}{4} m \cdot\left(\tau^{00}+i \pi\right) \cdot m} \theta_{\tau}\left(\bar{x}, \mu+\frac{i}{2 \pi} \tau^{00} \cdot m, x\right) .
\end{aligned}
$$

For a given function $\phi=\exp ($ if $) \in \mathscr{G}$ the vector part can be calculated from

to be

$$
\sum x_{k} \alpha_{k}=\sum \omega_{l}^{+} \int_{A_{l}^{+}} j(\phi)
$$

$$
x_{k}=\sum_{l} \tau_{k l}^{-+} \int_{A_{l}^{+}} j(\phi),
$$

whilst the winding numbers round the boundary are

$$
m_{k}=\int_{A_{k}^{0}} j(\phi) .
$$

(The generating function is supported on $\mathscr{T} \sigma$, so the winding number round $A_{0}$ is just the sum of $-m_{k}$.) We shall write $f_{e}$ for the exact part of $f$, that is $\phi_{e}=\exp \left(i f_{e}\right)$ is a function in $j^{-1}(\mathscr{V})$ such that $\phi / \phi_{e}$ is constant on $\mathscr{V} \times \mathscr{C}$-cosets. Finally we recall that the generating function is constant on $\mathscr{T}$ cosets, it depends only on the differences $\mu_{k}-\mu_{0}$ between values on $A_{k}^{0}$ and $A_{0}$, and these should replace $\mu$ in the earlier calculations.

Theorem 4.3. Let $\Omega_{\Upsilon}$ be the normalised fermion cyclic vector, for $\phi=\exp ($ if $) \in$ $\mathscr{G}$, let $m_{k}$ be the winding number round $A_{k}^{0}$, let $\mu_{k}$ and $\mu_{0}$ be its values at the base point $c_{k}$ on $A_{k}^{0}$ and $c_{0}$ on $A_{0}$, respectively, let

$$
x_{k}=\sum_{l} \tau_{k l}^{-+} \int_{A_{l}^{+}} j(\phi),
$$

and let $f_{e}$ be the exact part of $f$, and $f_{e}^{+}$denote the part which is holomorphic in $\Sigma_{+}$. Then the generating function is given by

$$
\begin{aligned}
\left\langle\Omega_{\Upsilon}, \Gamma(\phi) \Omega_{\Upsilon}\right\rangle= & \delta(m) e^{-\pi^{2} \bar{x}\left(\tau^{-+}\right)^{-1} x} e^{\frac{1}{4} m \cdot\left(\tau^{00}+i \pi\right) \cdot m} \frac{\theta_{\tau}\left(\bar{x}, \mu-\mu_{0}+\frac{i}{2 \pi} \tau^{00} \cdot m, x\right)}{\theta_{\tau}(0,0,0)} \\
& \times \exp \left(-\frac{1}{4} \int_{\partial \Sigma_{+}} f_{e} d f_{e}^{+}\right),
\end{aligned}
$$

where $\delta(m)$ is 1 when $m_{0}+m_{1}+\cdots+m_{p}=0$ and vanishes otherwise.

Remarks 4.2. This result should be compared with numerous papers in the physics literature [AMV, ANMV, ABNMV, E]. Mathematical gaps in these papers were filled for example in [KNTY,N]. It is important to note what is new about our approach. We have derived the generating functional and cyclic vector for both bosons and fermions from the underlying geometry. By contrast in [KNTY,N] the generating function for both bosons and fermions is postulated to be the same theta function (not proved) on the basis of earlier work of [DJKM] on hierarchies of soliton 
equations and tau functions. In this earlier work an algebraic approach to representations of Kac-Moody algebras in the language of formal infinite order differential operators (vertex operators) and power series is used (this framework is given mathematical precision in [KNTY,N]). This latter language cannot be readily related to the conventional one of representations on Hilbert spaces and with quantum field theories satisfying Wightman positivity. To see what is required in the genus zero case see [PS, CR]. An approach which is similar in spirit if not in detail to ours is contained in [R1].

We conclude by noting two consequences of our derivation.

Remarks 4.3. As a result of our convention that the cyclic vector should be fixed by $\mathscr{L}$, rather than having a more general eigenvalue, means that the theta function has characteristic $[0,0]$. In this connection it is interesting to notice that the CAR algebra depended only on the periodicity of the fermions on the boundary circles, and so did its representation. However, the Fock cyclic vector also depended on the choice of a compatible spin structure on the whole of $\Sigma$. It is interesting to note what whilst the lattice $\mathscr{L}$ preserves $\mathscr{K}_{-}$, the action of $\frac{1}{2} \mathscr{L}_{0}$ is locally holomorphic but changes some of the periods by half integers; it therefore maps elements of $\Gamma\left(\Sigma_{-}, \mathcal{O}(K)^{\frac{1}{2}}\right)$ to sections of another spin bundle. Accordingly it permutes the cyclic vectors for different spin structures. At the bosonic level, conjugation by elements of $\frac{1}{2} \mathscr{L}_{0}$ changes the characteristic of the theta function. In this way we see how the characteristics of the theta function are connected to the choice of spin bundle over the whole surface.

General theory tells us that the infinitesimal form of the boson generating function is given by

$$
\operatorname{tr}\left(P_{+} f P_{-} g P_{+}\right)=\left.\frac{\partial^{2}}{\partial s \partial t} F(\exp (i(s f+t g)))\right|_{s=t=0} .
$$

We have already remarked that the projections $P_{+}$and $P_{-}$are integral operators. Indeed $P_{+}$is defined by the Szegö kernel $\Lambda(x, \tilde{y})$, and $P_{-}$has the transpose kernel $\Lambda(y, \tilde{x}),[\mathrm{CHM}]$. We therefore see that

$$
\left(P_{+} f P_{-} g P_{+} \alpha\right)(w)=\int \Lambda(x, \tilde{y}) f(y) \Lambda(z, \tilde{y}) g(z) \Lambda(z, \tilde{w}) \alpha(w) .
$$

Taking the trace and simplifying we are left with

$$
\operatorname{tr}\left(P_{+} f P_{-} g P_{+}\right)=\int \Lambda(x, \tilde{y}) f(y) \Lambda(x, \tilde{y}) g(x)=\int \Lambda(x, \tilde{y})^{2} f(y) g(x) .
$$

Thus the kernel for the infinitesimal generating function should be $\Lambda(x, \tilde{y})^{2}$. According to formula 91 in $[\mathrm{H}]$ (or Corollary 2.12 in $[\mathrm{F}]$ ) this can be expressed as

$$
\Lambda(x, \tilde{y})^{2}=\mathscr{B}(x, \tilde{y})+\sum \frac{\partial^{2} \ln \theta}{\partial z_{k} \partial z_{l}} \omega_{k}^{r} \omega_{l}^{s},
$$

where the first term in this expression is the Bergmann kernel. This corresponds to the Gaussian term in the generating function $F$, whilst the remaining term is the infinitesimal part of the theta function arising from the globally holomorphic forms.

The simplicity of the eventual answer suggests that there should be an alternative derivation and we shall now sketch a more direct approach. The form of the fermion cyclic vector as a tensor product means that the generating function, $F$, is the 
product of its restrictions to $\mathscr{V}$ and $\mathscr{W}$. On $\mathscr{V}$, the generating function is simply the Gaussian appropriate to the Fock representation but on $\mathscr{Z} \times \mathscr{W} \times \mathscr{C}$, the situation is more complicated.

Our aim is to show that the generating function on the latter group is given by a theta function. To do this we need to derive some equivariance conditions from the existence of the lattice $\mathscr{L}$ found in Lemma 4.2. We first note that

$$
\begin{aligned}
\Gamma(\xi)^{*-1} \Psi(\alpha)^{*} \Gamma(\xi)^{*} & =\Psi(\xi \cdot \alpha)^{*} \\
& =\Psi(\tilde{\xi} \tilde{\alpha}),
\end{aligned}
$$

whence it follows that we may as well take

$$
\Gamma(\xi)^{*}=\Gamma(\tilde{\xi})^{-1} .
$$

Since $\Omega_{\Upsilon}$ is an analytic vector for the complexified action the generating function extends holomorphically to the complexification of $\mathscr{W}$. There is also a functional equation for $F$, resulting from the lattice action on $\Omega_{\Upsilon}$. In fact, since for $\lambda_{1}$ and $\lambda_{2}$ in $\mathscr{L}$ we have

$$
\left\langle\Gamma\left(\lambda_{1}\right) \Omega_{\Upsilon}, \Gamma(\xi) \Gamma\left(\lambda_{2}\right) \Omega_{\Upsilon}\right\rangle=\frac{\sigma\left(\xi, \lambda_{2}\right)}{\sigma\left(\tilde{\lambda}_{1}, \tilde{\lambda}_{1}^{-1} \xi \lambda_{2}\right)}\left\langle\Omega_{\Upsilon}, \Gamma\left(\tilde{\lambda}_{1}^{-1} \xi \lambda_{2}\right) \Omega_{\Upsilon}\right\rangle,
$$

we deduce that

$$
F(\xi)=\frac{\sigma\left(\xi, \lambda_{2}\right)}{\sigma\left(\tilde{\lambda}_{1}, \tilde{\lambda}_{1}^{-1} \xi \lambda_{2}\right)} F\left(\tilde{\lambda}_{1}^{-1} \xi \lambda_{2}\right)
$$

Thus the generating function satisfies an equivariance condition on $\mathscr{L} \tilde{\mathscr{L}}$ cosets.

Now we investigate the implications of (4.3). The action of $\mathscr{L}$ enables us to change the periods round $A^{0}, A^{-}$and $B^{-}$cycles by integers, so the action of $\tilde{\mathscr{L}}$ enables us to make integral changes to the values round $A^{0}, A^{+}$and $B^{+}$cycles. Now it is more convenient to think of such a lattice additively, that is to work with functions $f=\log (\xi)$. Such functions are defined only up to integer changes on the boundary components, and we have seen that these correspond to changes in the periods round $B^{0}$ cycles. (At first sight it appears that since boundary differences correspond to half the $B^{0}$ period it should be possible to make only even integer changes to these cycles. However, within $\mathscr{L}$ and so also in $\tilde{\mathscr{L}}$ the $B^{0}$ and $A^{0}$ periods are linked. By choosing elements of $\mathscr{L}$ and $\tilde{\mathscr{L}}$ so that the $A^{0}$ dependence cancels one finds that the $B^{0}$ changes can be integral.) Overall, then, the function $F$ is equivariant with respect to a lattice represented by integral changes to the periods round $A$ and $B$ cycles. By definition this lattice is defined by the period matrix, and by comparison with [M2] the functional equation is that for an even theta function. The generating function is therefore the product of a Gaussian and a theta function.

\section{The Boson Fermion Correspondence}

We have already noted in Corollary (2.2) that the fermion representation is equivalent to the tensor product of representations for the individual boundary circles capped by discs. For each such boundary component it is known that the fermion representation can be recovered from the boson operators. Let us choose an annular 
neighbourhood of a boundary circle and a local coordinate $z$ such that the boundary is $|z|=1$. Then following [S, $\mathrm{CH}, \mathrm{CR}]$ the fermions can be reconstructed using the "blips" $\gamma_{a} \in \mathscr{G}$, where $|a|<1$ and

$$
\gamma_{a}(z)=\left(\frac{\bar{a}}{\bar{a}}\right)^{\frac{1}{2}}\left(\frac{z-a}{z \bar{a}-1}\right) .
$$

In fact, it is known that for $\alpha$ concentrated on the annular neighbourhood of that boundary circle

$$
\Psi(\alpha)=\lim _{r \rightarrow 1} \int_{|a|=r} \alpha(a)\left(\left(1-|a|^{2}\right)^{-\frac{1}{2}} \Gamma\left(\gamma_{a}\right)\right) \sqrt{\frac{d a}{a}},
$$

where the limit means strong convergence on a dense domain (see [CR] for details). Note that since $\alpha$ is a half-form the factor $\sqrt{d a / a}$ turns this into a form which can then be integrated. We shall now show that these local blips can also be interpreted geometrically.

First let us consider the renormalisation factor $\left(1-|a|^{2}\right)^{-\frac{1}{2}}$. In terms of the local coordinate the prime form can be expressed as

$$
E(x, y)=\frac{y-x}{\sqrt{d x d y}}+O\left((x-y)^{3}\right),
$$

([F] Cor 2.5). The local expression for the involution is $\tilde{a}=1 / \bar{a}$, so that we have

$$
E(\tilde{a}, a)^{-1}=\frac{\sqrt{d(1 / \bar{a}) d a}}{\bar{a}^{-1}-a}=\frac{\sqrt{-d \bar{a} d a}}{\left(1-|a|^{2}\right)},
$$

whose square root is almost what we want.

Now this is not quite well defined in general, and one should rather use the expression

$$
\frac{i \theta(e-\tilde{a}+a)}{\theta(e) E(\tilde{a}, a)}
$$

for $e$ a half period fixed by the Schottky involution and having vanishing $B_{k}^{0} \cap \Sigma_{+}-$ components. This has the same limiting behaviour near the boundary and is, as Fay shows ([F] Cor 6.15 et seq), a positive section of the bundle $|K| \otimes(2 \operatorname{Re}(e))$, where $\operatorname{Re}(e)$ denotes the line bundle associated with the real part of $e$. Considering for the moment the case when $e=0$, we see that we may take a positive square root as a section of $|K|^{\frac{1}{2}}$. Since the boundary is oriented the restrictions of $|K|$ and $K$ there can be naturally identified. The general case of non-zero $e$ can be handled by multiplying through by an appropriate $\exp \left(-\sum e_{k} \int \omega_{k}^{0}\right)$ to convert it to the previous case. One then ends up with a square root which is a section of $|K|^{\frac{1}{2}} \otimes e$ restricted to an annular region containing the boundary circle. It converges to the half form $(i d \theta)^{\frac{1}{2}}=(d w / w)^{\frac{1}{2}}$ on the circle. It follows that in the annular region $\alpha(a) E(\tilde{a}, a)^{-\frac{1}{2}}$ is a one-form and we have:

$$
\Psi(\alpha)=\lim _{\lambda \rightarrow 1} \int_{|a|=\lambda} \alpha(a) E(\tilde{a}, a)^{-\frac{1}{2}} \Gamma\left(\gamma_{a}\right) .
$$


To interpret the blip $\gamma_{a}$ we recall that there is a family of distinguished meromorphic functions on $\Sigma_{+}$whose values on $\partial \Sigma_{+}$have modulus 1 and with the minimal number of zeroes, [F] Theorem 6.6. Tailoring the result to our needs, for $a \in \Sigma_{+}$ and $s$ a suitable even half period we take

$$
\varepsilon_{a}(z)=\frac{\theta(z-\tilde{a}-s)}{\theta(z-a-s)} \frac{E(z, a)}{E(z, \tilde{a})} \exp \left(\frac{1}{2} \sum_{1}^{p} \mu_{j} \int_{a}^{\tilde{a}} \omega_{j}\right),
$$

where $1+\mu_{j}$ agrees modulo 2 with the winding number of $\varepsilon_{a}$ round the $j^{\text {th }}$ boundary component $\partial_{j} \Sigma_{+}$. Since $\varepsilon_{a}$ has modulus 1 on the boundary circles it represents an element of $\mathscr{G}$.

Lemma 5.1. In sufficiently small annular neighbourhoods of the boundary circles the function $\gamma_{a}^{-1} \varepsilon_{a}$ is defined and converges pointwise as $a \rightarrow w$ on the unit circle to the constant function 1.

Proof. That a choice of annular region exists is clear from the discussion at the beginning of this section. As $a \rightarrow w$ on the unit circle so does $\tilde{a}$, and since the theta functions are non-singular in some neighbourhood of the circle the first ratio in (5.2) tends to 1 . The integral in the exponential in (5.2) tends to 0 giving another unit factor. However, if $z, a$ and $\tilde{a}$ are all close to a single boundary circle then the prime forms can have singularities. Locally one has

$$
\frac{E(z, a)}{E(z, \tilde{a})}=\left(\frac{z-a}{z-1 / \bar{a}}\right)\left(\frac{d(1 / \bar{a})}{d a}\right)^{\frac{1}{2}}=\left(\frac{z-a}{z \bar{a}-1}\right)\left(\frac{-d \bar{a}}{d a}\right)^{\frac{1}{2}} \text {. }
$$

As $a \rightarrow w=e^{i \theta}$ the derivative $-d \bar{a} / d a \rightarrow e^{-2 i \theta}=\bar{w} / w$, so that locally

$$
\varepsilon_{a}=\left(\frac{\bar{a}}{a}\right)\left(\frac{z-a}{\bar{a} z-1}\right)=\gamma_{a} .
$$

If $z$ is on a different boundary component to $w$ then the ratio of the prime forms also converges to 1 , so that on the other boundary circles $\varepsilon_{a} \rightarrow 1$.

There is a more sophisticated way of arriving at the same result by fixing some $c \in \Sigma_{+}$and using the function $\varepsilon_{c}$ to uniformize $\Sigma$ by mapping it to the Riemann sphere. Then using the addition formula, $[\mathrm{F}]$, Eq. 45 , we calculate that

$\frac{\varepsilon_{c}(z)-\varepsilon_{c}(a)}{\varepsilon_{c}(z)-\varepsilon_{c}(\tilde{a})}=\frac{\theta(z+a-c-\tilde{c})}{\theta(z+\tilde{a}-c-\tilde{c})} \frac{\theta(\tilde{a}-c)}{\theta(a-c)} \frac{\theta(z-a)}{\theta(z-\tilde{a})} \frac{E(\tilde{a}, \tilde{c})}{E(a, \tilde{c})} \exp \left(-\frac{1}{2} \sum \mu_{j} \int_{a}^{\tilde{a}} \omega_{j}\right) \varepsilon_{a}$.

All the factors on the right-hand side except $\varepsilon_{a}$ are close to 1 , so that $\varepsilon_{a}$ is approximately the same as the cross ratio on the left-hand side which is just the standard blip expressed using $\varepsilon_{c}$ as a coordinate.

One way to interpret this lemma is that as $a \mapsto w$ on the unit circle the meromorphic function $\varepsilon_{a}$ on the Riemann surface approaches a (singular) distribution. Recalling Segal's blip construction of vertex operators (see [PS]) this suggests how to construct regularised "vertex operators" on the Riemann surface which give a precise analytic meaning to the boson-fermion correspondence.

Theorem 5.2. For $\Phi$ in a dense domain of the Fock space

$$
\Psi(\alpha) \Phi=\lim _{\lambda \rightarrow 1} \int_{|a|=\lambda} \alpha(a)\left(E(\tilde{a}, a)^{-\frac{1}{2}} \Gamma\left(\varepsilon_{a}\right)\right) \Phi .
$$


Remarks 5.1. This theorem may be interpreted as saying that the Segal vertex operator on a Riemann surface is given by $\Gamma\left(\varepsilon_{a}\right)$.

Proof. For a precise description of the dense domain of convergence we refer to [CR]. This latter paper and our remarks above imply that it is sufficient to show that the operator $\Gamma\left(\varepsilon_{a}\right) \Gamma\left(\gamma_{a}\right)^{-1}$ converges in norm to the identity on the Fermion Fock space as $a \rightarrow w$ on the unit circle. Let $Q_{ \pm}$denote the standard Hardy space projections on $L^{2}\left(S^{1}\right)$. Then, as the fermion representation is equivalent to that defined by this Hardy space decomposition it is sufficient by [CR] to prove that $\eta_{a}=\varepsilon_{a} \gamma_{a}^{-1}$ converges uniformly to the function which is identically one on the unit circle and that $Q_{ \pm} \eta_{a} Q_{\mp}$ converges to zero in Hilbert-Schmidt norm on operators on $L^{2}\left(S^{1}\right)$. Now by [F, Cor. 2.5] we have $\eta_{a}=f(z-a) / f(z-\tilde{a})$, where $f$ is analytic on a neighbourhood of the unit disc $\{z|| z \mid \leqq 1\}$. It is then easy to show that the desired result follows by proving for $f_{a}(z)=f(z-a)$ that $f_{a}-f_{\tilde{a}}$ converges uniformly on the unit circle to zero and that $Q_{ \pm}\left(f_{a}-f_{\tilde{a}}\right) Q_{\mp}$ converges in Hilbert-Schmidt norm to zero. Now Hilbert-Schmidt convergence of such an operator is equivalent (see [CR]) to convergence of $f_{a}-f_{\tilde{a}}$ to zero in $H^{1 / 2}$ norm as $a \rightarrow w$ in the unit circle. It suffices therefore to prove that both $f_{a}-f_{\tilde{a}}$ and its derivative converge to zero uniformly on the unit circle. But the families of functions $\left\{f_{a}\right\},\left\{f_{\tilde{a}}\right\}$, and the derivatives, $\left\{f_{a}^{\prime}\right\}$ and $\left\{f_{\tilde{a}}^{\prime}\right\}$, are each equicontinuous for $a$ in an annulus about the unit circle. Consider the first family of functions. Equicontinuity implies that uniformly convergent subsequences of $\left\{f_{a}\right\}$ exist. As $f_{a}$ converges pointwise to $f_{w}$ as $a \rightarrow w$, all uniformly convergent subsequences indexed by a sequence $a_{n} \rightarrow w$ as $n \rightarrow \infty$, must satisfy $f_{a_{n}} \rightarrow f_{w}$ uniformly. That is all uniformly convergent subsequences have the same limit and hence a standard argument by contradiction now shows that $f_{a}-f_{w}$ itself converges uniformly to zero as $a \rightarrow w$. Similar statements hold for the other families of functions proving the desired uniform convergence.

\section{Coverings}

From a physical point of view conformal field theories represent a quantum analogue of two-dimensional hydrodynamics. In that classical theory conformal mappings between Riemann surfaces play an important role, as interesting problems often simplify when transformed. For example, the Joukowski transformation $z=$ $w+c^{2} / w$ maps the flow round an aerofoil into that round a disc. In quantum theory the exponential map $z=\exp (w / \beta)$ takes a zero temperature system into one at inverse temperature $\beta$, or alternatively takes an infinite to a finite system. An account of various physical applications is given in the survey article by Cardy, [C]. There are mathematical reasons too for being interested in coverings. It is known that generically every vector bundle over a Riemann surface is the image of a line bundle over some covering surface [AB]. The use of coverings therefore permits one to extend the results of the previous sections to vector-valued spinors and loop groups of mappings into unitary and other groups.

The recent article $[\mathrm{AB}]$ discusses the geometric theory in the context of integrable models. We shall quickly review some of the features of finite coverings in 
our setting. (The Joukowski transformation can be rewritten in the form

$$
\left(\frac{z-2 c}{z+2 c}\right)=\left(\frac{w-c}{w+c}\right)^{2}
$$

and so provides an interesting example of such a covering.)

Consider a map $f$ from a Riemann surface $\hat{X}$ to $X$. If $L$ is a line bundle over $\hat{X}$ then the sections, $\Gamma(\hat{X}, \mathcal{O}(L))$ form a module for germs of holomorphic functions $\mathcal{O}_{\hat{X}}$ on $\hat{X}$. If $f^{*}$ denotes the map from holomorphic germs $\mathcal{O}_{X}$ on $X$ to $\mathcal{O}_{\hat{X}}$ obtained by composing with $f$, then we may also regard $\Gamma(\hat{X}, \mathcal{O}(L))$ as an $\mathcal{O}_{X}$-module. In fact it can be identified with sections of a vector bundle over $X$.

For simplicity let us assume that both surfaces admit coordinates, $w$ and $z$, say, that $f$ is a polynomial of degree $N$ and write $z=f(w)$. The fibre of $L$ at $w \in \hat{X}$ can then be identified with

$$
\Gamma(\hat{X}, \mathcal{O}(L)) / w \Gamma(\hat{X}, \mathcal{O}(L)),
$$

and the push-down vector bundle over $X$ has fibre at $z=f(w) \in X$

$$
V_{f}=\Gamma(\hat{X}, \mathcal{O}(L)) / f(w) \Gamma(\hat{X}, \mathcal{O}(L)) .
$$

Generically there are $N$ distinct points $v$ such that $f(v)=z$ and the $N$-dimensional space $V_{f}$ can be identified with the direct sum of the $N$ lines $L_{v}$.

The relation between sections of $L$ and of $V$ can easily be described explicitly by using the Lagrange interpolation polynomial

$$
p_{w}(v)=\frac{f(w)-f(v)}{(w-v) f^{\prime}(w)},
$$

which takes the value 1 when $v=w$ and vanishes for other $v$ satisfying $f(v)=f(w)$. Writing $p_{w}$ explicitly as a polynomial

$$
p_{w}(v)=\sum_{j=1}^{N} p_{j}(w) v^{j},
$$

we can expand any function $\psi$ on $\hat{X}$ as

where

$$
\psi(w)=\sum_{j=1}^{N} p_{j}(w) \psi_{j}(f(w)),
$$

$$
\psi_{j}(z)=\sum_{v \in f^{-1}(z)} v^{j} \psi(v)
$$

In this picture multiplication by a scalar function $\phi$ on $\hat{X}$ can be represented by multiplication by matrix-valued function on $X$. In fact

$$
\begin{aligned}
(\phi \cdot \psi)(z) & =\sum_{v \in f^{-1}(z)} v^{j} \phi(v) \psi(v)=\sum_{v \in f^{-1}(z)} v^{j} \phi(v) \sum_{k=1}^{N} p_{k}(v) \psi_{k}(f(v)) \\
& =\sum_{k=1}^{N} \phi_{j k}(z) \psi_{k}(z)
\end{aligned}
$$

where

$$
\phi_{j k}(z)=\sum_{v \in f^{-1}(z)} v^{j} \phi(v) p_{k}(v)
$$


In particular when $\phi=f$ we have

$$
f_{j k}(z)=\sum_{v \in f^{-1}(z)} v^{j} f(v) p_{k}(v)=z \sum v^{j} p_{k}(v)=z \delta_{j k} .
$$

This in turn means that if $W$ is the matrix representing multiplication by $w$ then $f(W)=z 1$.

Rather than direct use of the formulae, however, it is more efficient to write $f$ as a product of factors $f_{j}(z)=\left(z-a_{j}\right)^{N_{j}}$ and exploit the primary decomposition theorem to obtain

$$
V_{f} \cong V_{f_{1}} \oplus V_{f_{2}} \oplus \cdots \oplus V_{f_{r}}
$$

This enables us to concentrate on the special case when $f(z)=z^{N}$, where $p_{j}(w)=w^{-j} / N$, so that

$$
\psi(w)=\frac{1}{N} \sum_{j} w^{-j} \psi_{j}\left(w^{N}\right) .
$$

When $L$ is the line bundle of half-forms, the inner product can also easily be computed. In the simplest case, when the boundary is given by a level curve of $|w|$, we have

$$
\begin{aligned}
\langle\alpha, \beta\rangle & =\int_{\partial \hat{X}} \bar{\alpha}(w) \beta(w)=\frac{1}{N^{2}} \int_{\partial \hat{X}} \sum_{j, k} \bar{w}^{-j} w^{-k} \tilde{\alpha}_{j}\left(w^{N}\right) \beta_{k}\left(w^{N}\right) \\
& =\frac{1}{N^{2}} \int_{\partial \hat{X}} \sum_{j, k} w^{j-k} \tilde{\alpha}_{j}\left(w^{N}\right) \beta_{k}\left(w^{N}\right)=\frac{1}{N} \sum_{k} \int_{\partial \hat{X}} \tilde{\alpha}_{k}\left(w^{N}\right) \beta_{k}\left(w^{N}\right) .
\end{aligned}
$$

Now the boundary of $\hat{X}$ covers $N$ times that of $X$, so that

$$
\langle\alpha, \beta\rangle=\sum_{k} \int_{\partial X} \tilde{\alpha}_{k}\left(w^{N}\right) \beta_{k}\left(w^{N}\right),
$$

which is what one would expect for a multicomponent fermion theory. Summarising we have the theorem.

Theorem 6.1. The fermion theories for sections of $L$ over $\hat{X}$ and for sections of $V$ over $X$ are equivalent under the above correspondence.

Returning to the case of general $L$ but with $z=w^{N}$, the bosonic action is then given by the matrix

$$
\phi_{j k}(z)=\frac{1}{N} \sum_{v \in f^{-1}(z)} v^{j-k} \phi(v) .
$$

In particular, taking $\phi(w)=w$ we get the matrix

$$
W_{j k}=\frac{1}{N} \sum v^{j-k+1}
$$

which is 1 for $k=j+1, z$ for $k=N-1, j=0$, and otherwise vanishes. That is

$$
W=\left(\begin{array}{ccccc}
0 & 0 & \ldots & 0 & z \\
1 & 0 & \ldots & 0 & 0 \\
0 & 1 & \ldots & 0 & 0 \\
\vdots & & & & \vdots \\
0 & 0 & \ldots & 1 & 0
\end{array}\right)
$$


which is essentially the formula of $[\mathrm{AB}]$, Sect. 4. The general case is given by a direct sum of such matrices, one corresponding to each primary factor of the covering map, $f$.

One can already detect some features of the covering on $\hat{X}$ if knows the action of the deck transformation group. For $f(w)=w^{N}$, one may cyclicly permute the roots, or equivalently multiply them by powers of $\lambda=\exp (2 \pi i / N)$. Setting $(S \psi)(w)=$ $\psi(\lambda w)$ we have

$$
(S \psi)_{j}(w)=\sum_{x} x^{j}(S \psi)(x)=\sum x^{j} \psi(\lambda x)=\lambda^{-j}(S \psi)_{j}(z)
$$

so that $S$ has the matrix

$$
S=\left(\begin{array}{cccc}
1 & 0 & \ldots & 0 \\
0 & \lambda & \ldots & 0 \\
\vdots & & & \vdots \\
0 & 0 & \ldots & \lambda^{N-1}
\end{array}\right)
$$

The two matrices $\sigma$ and $W$ satisfy the relation

$$
S W=\lambda W S,
$$

and so generate a projective representation of the finite group $\mathbf{Z}_{N} \times \mathbf{Z}_{N}$. For $z \neq 0$ this representation is irreducible and so generates the whole general linear group, but at the ramification point $z=0$ we have $W^{N}=0$, so that $W$ is nilpotent and the representation is no longer irreducible though still indecomposible.

The significance of this can be seen by considering the covering of the annulus $q^{2} \leqq|w| \leqq 1$ defined by $\sqrt{z-q}$, in other words the covering given by $z=w^{2}+q$. The covering space has three boundary circles, two of which cover one boundary of the annulus and one doubly covers the other end. Line bundles over the covering give rank 2 vector bundles over the annulus, and the nilpotent matrix $W$ allows one to patch the rather different behaviour at the two ends of the annulus.

The other feature of above discussion which is typical is the fact that the gauge transformations and deck group give all linear transformations of the vector space at $z$.

Theorem 6.2. If the deck group $D$ acts transitively on the sheets of the covering then the algebra generated by $D$ and $\operatorname{Map}(\partial X, \mathbf{T})$ is the algebra generated by $\operatorname{Map}(\partial X, \mathbf{U}(N))$.

Proof. The algebra generated by $D$ and $\operatorname{Map}(\partial \hat{X}, \mathbf{T})$ is

$$
\left\langle D, \operatorname{Map}((\partial \hat{X}, \mathbf{T})\rangle^{\prime \prime}=\left(D^{\prime} \cap \operatorname{Map}(\partial \hat{X}, \mathbf{T})^{\prime}\right)^{\prime} .\right.
$$

Now, acting on the fermion space, the commutator of $\operatorname{Map}(\partial \hat{X}, \mathbf{T})$ is $\operatorname{Map}(\partial \hat{X}, \mathbf{C})$, and its inter-section with $D^{\prime}$ consists of the $D$-invariant elements. Thus we have

$$
\begin{aligned}
\left(D^{\prime} \cap \operatorname{Map}(\partial \hat{X}, \mathbf{T})^{\prime}\right) & =\operatorname{Map}(\partial \hat{X}, \mathbf{C})^{D}=\operatorname{Map}(\partial \hat{X} / D, \mathbf{C}) \\
& =\operatorname{Map}(\partial X, \mathbf{C}) .
\end{aligned}
$$

The commutant of this is the whole of $\operatorname{Map}(\partial X, \mathbf{G L}(N))$ which in turn is also the algebra generated by $\operatorname{Map}(\partial X, \mathbf{U}(N))$, as claimed. 
As with the fermion inner product, since the boson multiplier is given in a coordinate-free form and all the constructions are natural it can easily be computed for the multi-component boson theory. The boson theory is nonetheless more subtle than that of the fermions as the following outline example shows.

It is common in the theory of loop groups to consider maps into the semisimple group, $\mathbf{S U}(N)$, rather than into the reductive group, $\mathbf{U}(N)$, [PS]. In terms of an $N$-fold covering, the Cartan subgroup is generated by subgroups $\mathscr{T}_{j k}$ consisting of functions which multiply by $\phi$ on the $j^{\text {th }}$ sheet and by $\phi^{-1}$ on the $k^{\text {th }}$ sheet. (In practice one would have to regard these as limits of smoothed versions to avoid discontinuities at the cuts.) For each such subgroup of functions one can consider the blips $\phi=\varepsilon_{a}(z)$. Since $\varepsilon_{a}$ is essentially the exponential of a reproducing kernel, it is the product of the corresponding functions $\varepsilon_{b}(w)$ as $b$ runs over the $N^{\text {th }}$ roots of $a$. On the $j^{\text {th }}$ sheet, the function $\varepsilon_{b_{j}}$ corresponding to the root $b_{j}$ in that sheet is of special interest, but all the other factors, corresponding to $b$ in other sheets are nearly constant, and the corresponding element on the $k^{\text {th }}$ sheet is $\varepsilon_{b_{k}}$. To a good approximation the blip in the subgroup $\mathscr{T}_{j k}$ is the product $\varepsilon_{b_{j}} \varepsilon_{b_{k}}^{-1}$. Now the limit of the appropriately renormalised operator coming from the boson representation of $\varepsilon_{b_{j}}$ is the unsmeared fermion operator $\Psi_{b_{j}}$, and similarly the renormalised limit of the operator representing $\varepsilon_{b_{k}}^{-1}$ is $\Psi_{b_{k}}^{*}$, so that, by taking the square of the normal convergence factor (one copy for each of the two $\varepsilon_{b}$ ) we get a collection of operators which "coverges" to a renormalised product $\Psi_{b_{j}} \Psi_{b_{k}}^{*}$ of unsmeared fermion operators concentrated at $b_{j}$ and $b_{k}$. But this product is precisely the current corresponding to an element in the Lie algebra of $\operatorname{Map}(\partial X, \mathbf{S U}(N))$ taking values in the $(j, k)^{\text {th }}$ root vector. In other words the analogue of the procedure which reconstructed the fermion operators from the bosons, this time reconstructs the off-diagonal elements of $\operatorname{Map}(\partial X, \mathbf{s u}(N))$ from its diagonal Cartan subgroup. Although the above argument is totally non-rigorous, the conclusion is known to be correct for unramified coverings, $[\mathrm{S} 1]$ and the covering space ideas provide a new perspective on it.

\section{KMS Type Conditions}

Let us now restrict to the subgroup $\mathscr{G}_{1}$ of functions which are identically 1 on the base circle $A_{0}$. (One could argue similarly for functions which take the value 1 on several boundary circles.) For $\xi$ and $\eta$ in $\mathscr{G}_{1}$ we consider $\langle\Omega, \Gamma(\xi) \Gamma(\eta) \Omega\rangle$. Suppose that there is a function $\xi_{+}$holomorphic on $\Sigma_{+}$whose boundary values agree with $\xi$ except possibly on the base circle. Then $\tilde{\xi}_{+}$is holomorphic on $\Sigma_{-}$and it follows from the bosonic characterisation of the fermion cyclic vector that

$$
\begin{aligned}
\langle\Omega, \Gamma(\xi) \Gamma(\eta) \Omega\rangle & =\left\langle\Gamma\left(\tilde{\xi}_{+}\right) \Omega, \Gamma(\xi) \Gamma(\eta) \Omega\right\rangle=\left\langle\Omega, \Gamma\left(\tilde{\xi}_{+}^{-1}\right) \Gamma(\xi) \Gamma(\eta) \Omega\right\rangle \\
& =\sigma\left(\tilde{\xi}_{+}, \tilde{\xi}_{+}^{-1} \xi\right)^{-1}\left\langle\Omega, \Gamma\left(\tilde{\xi}_{+}^{-1} \xi\right) \Gamma(\eta) \Omega\right\rangle .
\end{aligned}
$$

Now $\tilde{\xi}_{+}^{-1} \xi$ and $\eta$ have disjoint supports and so commute. We therefore deduce that

$$
\langle\Omega, \Gamma(\xi) \Gamma(\eta) \Omega\rangle=\sigma\left(\tilde{\xi}_{+}, \tilde{\xi}_{+}^{-1} \xi\right)^{-1}\left\langle\Omega, \Gamma(\eta) \Gamma\left(\tilde{\xi}_{+}^{-1} \xi\right) \Omega\right\rangle .
$$

Next suppose that there is a function $\xi_{-}$holomorphic on $\Sigma_{-}$which agrees with $\xi^{-1} \tilde{\xi}_{+}$on the base circle. Then $\tilde{\xi}=\tilde{\xi}_{+}^{-1} \xi \xi_{-}$is in $\mathscr{G}_{1}$ and is the analytic continuation 
of $\xi$ round $\Sigma$, and also

$$
\begin{aligned}
\langle\Omega, \Gamma(\xi) \Gamma(\eta) \Omega\rangle & =\sigma\left(\tilde{\xi}_{+}, \tilde{\xi}_{+}^{-1} \xi\right)^{-1}\left\langle\Omega, \Gamma(\eta) \Gamma\left(\tilde{\xi}_{+}^{-1} \xi\right) \Gamma\left(\xi_{-}\right) \Omega\right\rangle \\
& =\frac{\sigma\left(\tilde{\xi}_{+}^{-1} \xi_{,} \xi_{-}\right)}{\sigma\left(\tilde{\xi}_{+}, \tilde{\xi}_{+}^{-1} \xi\right)}\langle\Omega, \Gamma(\eta) \Gamma(\hat{\xi}) \Omega\rangle
\end{aligned}
$$

Summarising this we have the following theorem.

Theorem 7.1. Let $\xi, \hat{\xi}$ and $\eta$ be elements of the group $\mathscr{G}_{1}$ of functions which are identically 1 on $A_{0}$, and suppose that there is a holomorphic function on $\Sigma$ cut along the $A^{0}$ cycles other than $A_{0}$ whose boundary values agree at one end with $\xi$ and at the other with $\hat{\xi}$. Then

$$
\langle\Omega, \Gamma(\xi) \Gamma(\eta) \Omega\rangle=\frac{\sigma\left(\tilde{\xi}_{+}^{-1} \xi, \xi_{-}\right)}{\sigma\left(\tilde{\xi}_{+}, \tilde{\xi}_{+}^{-1} \xi\right)}\langle\Omega, \Gamma(\eta) \Gamma(\hat{\xi}) \Omega\rangle,
$$

where $\xi_{ \pm}$are the interpolating functions in $\Sigma_{ \pm}$.

This identity for the two point correlation function generalises the standard KMS condition on the torus [CH1]. When $\Sigma_{+}$is an annulus, $\mathscr{G}_{1}$ consists of functions on the single non-base boundary circle. The real line acts as automorphisms by rotation of that circle. On a dense subset of the functions this automorphism extends holomorphically around the torus which is the Schottky double of the annulus to give a rotation translated by $i \tau$, where $\tau$ is the complex number which gives the period matrix in this case. Thus $\hat{\xi}$ is the image of $\xi$ under the complexified rotation through $i \tau$ and the identity reduces to the standard KMS identity.

The same identity can also be interpreted in several other ways. For example, if $\Sigma$ has genus larger than 1 then the uniformisation theorem tells us that $\Sigma$ can be identified with the quotient of a standard disc by the action of a discrete group $\Delta$. Functions of $\Sigma$ lift to equivariant functions on the disc. If we look at a particular sheet then the analytic continuation of $\xi$ round $\Sigma$ results in its image under the appropriate element of $\Delta$, in fact the element given by hyperbolic reflection in the arc which covers the base circle in this particular sheet.

One can also give the identity a more algebraic flavour by noting that $\mathbf{T}^{p}$ acts as automorphisms of $\mathscr{G}_{1}$ by rotating the $p$ non-base boundary circles. However we will not pursue this here.

\section{References}

[AB] Adams, M.R., Bergvelt, M.J.: The Krichever map, vector bundles over algebraic curves, and Heisenberg algebras. Commun. Math. Phys. 154, 265-305 (1993)

[AMV] Alvarez-Gaumé, L., Moore, G., Vafa, C.: Theta functions, modular invariance, and strings. Commun. Math. Phys. 106, 1-40 (1986)

[ANMV] Alvarez-Gaumé, L., Nelson, P., Moore, G., Vafa, C.: Bosonization in arbitrary genus. Phys. Lett. B178, 41 (1986)

[ABNMV] Alvarez-Gaumé, L., Bost, J.-B., Nelson, P., Moore, G., Vafa, C.: Bosonization on higher genus Riemann surfaces. Commun. Math. Phys. 112, 503-552 (1987)

[Ar] Araki, H.: Bogoliubov automorphisms and Fock representations of canonical anticommutation relations. Contemp. Math. 62, 23-141 (1987) 
[C] Cardy, J.L.: Conformal invariance. Phase transitions and critical phenomena, 11, London: Academic Press, 1987, pp. 55-126

[CEH] Carey, A.L., Eastwood, M.G., Hannabuss, K.C.: Riemann surfaces, Clifford algebras and infinite dimensional groups, Commun. Math. Phys. 130, 217-236 (1990)

[CH1] Carey, A.L., Hannabuss, K.C.: Some simple examples of conformal field theories, Int. J. Mod. Phys. B4, 1059-1068 (1990)

[CH2] Carey, A.L., Hannabuss, K.C.: Temperature states on loop groups, theta functions and the Luttinger model, J. Funct. Anal. 75, 128-160 (1987)

[CHM] Carey, A.L., Hannabuss, K.C., Murray, M.K.: Free fermions on Riemann surfaces and spectral curves of the chiral Potts model. Proceedings of the 2nd International Conference on Topological Methods in Quantum Field Theory, Turku, Finland, 1991, Singapore: World Scientific, 1992

[CHMS] Carey, A.L., Hannabuss, K.C., Mason, L.J., Singer, M.A.: The Landau-Lifshitz equation, elliptic curves and the Ward transform, Commun. Math. Phys. 154, 25-47 (1993)

[CP] Carey, A.L., Palmer, J.: Infinite complex spin groups. J. Funct. Anal. 83, 1-43 (1989)

[CR] Carey, A.L., Ruijsenaars, S.N.M.: On fermion gauge groups, current algebras and KacMoody algebras. Acta Applic. Math. 10, 1-86 (1987)

[DJKM] Date, E., Jimbo, M., Kashiwara, M., Miwa, T.: Transformation groups for soliton equations. Proceedings of RIMS symposium. Ed. Jimbo, M., Miwa, T., Singapore: World Scientific, 1983, pp. 39-120

[E] Eguchi, T.: Chiral bosonization on a Riemann surface. Proceedings of the First Asia Pacific Workshop on High Energy Physics, "Conformal Field Theory, Anomalies and Super Strings"; Ed. Baaquie, B.E. et al, Singapore: World Scientific, 1987

[F] Fay, J.D.: Theta functions on Riemann surfaces. Springer Lecture Notes in Mathematics 352, 1973

[H] Hejhal, D.A.: Theta functions, kernel functions and Abelian integrals. Mem. Am. Math. Soc. 129, 1972

[JKL] Jaffe, A., Klimek, S., Lesniewski, A.: Representations of the Heisenberg algebra on a Riemann surface. Commun. Math. Phys. 126, 421-431 (1989)

[KNTY] Kawamoto, N., Namikawa, Y., Tsuchiya, A., Yamada, Y.: Geometric realization of conformal field theory on Riemann surfaces. Commun. Math. Phys. 116, 247-308 (1988)

[M1] Mackey, G.W.: Unitary representations of group extensions I. Acta Math. 99, 265-311 (1958)

[M2] Mackey, G.W.: Induced representations of locally compact groups and applications. In: Functional Analysis and related fields, (ed. F.E. Browder), Berlin: Springer Verlag, 1970

[N] Namikawa, Y.: A conformal field theory on Riemann surfaces realized as quantized moduli theory on Riemann surface. Pro. Symp. Pure Math. 49, (1989)

[PS] Pressley, A.N., Segal, G.B.: Loop groups. Oxford: Oxford University Press, 1986

[R1] Raina, A.K.: Fay's trisecant identity and Wick's theorem: An algebraic geometry viewpoint. Expos. Math. 8, 227-245 (1990)

[R2] Raina, A.K.: An algebraic geometry study of currents in the $b$-c-system. Preprint TIFR/TH/92-68 (1992)

[S1] Segal, G.B.: Unitary representations of some infinite-dimensional groups. Commun. Math. Phys. 80, 301-42 (1981)

[S2] Segal, G.B.: The definition of conformal field theory. (draft of paper)

[SW] Segal, G.B., Wilson, G.: Loop groups and equations of KdV type. Publ Math IHES 61, 5-65 (1989) 
